\title{
A VARIFOLD SOLUTION TO THE NONLINEAR EQUATION OF MOTION OF A VIBRATING MEMBRANE
}

\author{
By Daisuke Fujiwara and Shoichiro Takakuwa
}

\section{$\S 1$. Introduction.}

Let $\Omega$ be a bounded domain in $\boldsymbol{R}^{n}$ with the boundary $\partial \Omega$ which is a Lipschitz manifold. Then the equation of motion of a vibrating membrane is as follows:

$$
D_{t}^{2} u(t, x)-\sum_{\jmath=1}^{n} D_{j}\left\{D_{\jmath} u(t, x)\left(1+|D u(t, x)|^{2}\right)^{-1 / 2}\right\}=0, \quad x \in \Omega,
$$

where $D_{t}$ denotes $\partial / \partial t$ and $D_{\jmath}$ denotes $\partial / \partial x_{\jmath}, j=1,2, \cdots, n$. The initial and the boundary conditions we shall consider are

$$
\begin{gathered}
u(0, x)=u_{0}(x), \quad D_{t} u(0, x)=u_{1}(x), \\
u(t, x)=0 \quad \text { for } x \text { in } \partial \Omega .
\end{gathered}
$$

If $u_{0}(x)$ and $u_{1}(x)$ are sufficiently smooth, there exists a unique genuine solution of (1.1), (1.2) and (1.3) for a short time interval. (cf. Kato [9] and Shibata-Tsutsumi [10]). On the other hand, existence global in time of even a weak solution is not proved in the case $n>1$.

The purpose of the present paper is to treat the above equation by virtue of the theory of varifolds introduced by Almgren Jr. [2]. A varifold is a generalization of the notion of a function and was successfully used in the direct approach of the Plateau's problem. We shall define a generalized solution of the equation (1.1) in terms of varifolds, which we call the varifold solution. And we shall prove existence, global in time, of a varifold solution of (1.1), (1.2) and (1.3). Thus this paper is closely related with the works of Tartar [11], [12] and that of DiPerna [5].

Although a varifold solution is quite a weak notion, it satisfies a generalization of the Hamilton's principle:

$$
\delta \int_{0}^{T} d t \int_{\Omega}\left\{\frac{1}{2}\left|D_{t} u(t, x)\right|^{2}-\left(1+|D u(t, x)|^{2}\right)^{1 / 2}\right\} d x=0
$$

under appropriate assumptions.

Before introducing a varifold solution, we shall formulate, in $\S 2$, the notion

Received April 1, 1985 
of a weak solution of (1.1) in terms of functions of bounded variations of $n$-variables. (cf. De Giorgi [4] and Giusti [8]). This is interesting in itself and will help us to treat varifold solutions.

$\S 3$ is devoted to the definition of the notion of a varifold solution of (1.1).

In $\S 4$ we prove existence, global in time, of a varifold solution of (1.1), (1.2) and (1.3). This is done by the Ritz-Galerkin approximation method.

In $\S 5$ we show that the approximating sequence of Ritz-Galerkin method coverges to a function $u(t, x)$ of bounded variation in $x$.

In $\S 6$, we shall prove that the global varifold solution can be identified with $u(t, x)$ if $u(t, x)$ satisfies the energy conservation law. This will be done in Theorem 4.

A generalization of Hamilton's principle is proved in $\S 7$.

\section{Table of contents}

$\S 1$. Introduction.

$\S 2$. A weak solution.

$\S 3$. Definition of a varifold solution.

$\S 4$. Existence of a global varifold solution.

$\S 5$. Convergence in the space of BV-functions.

$\S 6$. The varifold solution and the BV-function.

$\S 7$. Generalized Hamilton's principle.

\section{Notations.}

The following usual notations are used: If $x$ and $y$ are two vectors in $\boldsymbol{R}^{k}$, $x \cdot y$ is the Euclidean inner product of $x$ and $y$, and $|x|$ is the length of $x$. If $M$ is a Radon measure on a $\sigma$-compact metric space $X$ and $\psi$ is a continuous function on $X$ then

$$
\langle M, \phi\rangle=\int_{X} \phi(x) d M(x)
$$

and spt $M$ is the support of $M . \mathscr{H}_{n}$ denotes the Hausdorff measure of dimension $n$. Let $m \geqq 0$ be an integer. Then

$\mathcal{C}^{m}(\Omega)$ denotes the space of functions of class $\mathcal{C}^{m}$ in $\Omega$.

$\mathcal{C}_{0}^{m}(\Omega)=\left\{u \in \mathcal{C}^{m}(\Omega)\right.$; spt $u$ is compact $\}$.

If $Y$ is a topological vector space and $U$ is an open subset of $\boldsymbol{R}^{k}$,

$\mathcal{C}^{m}(U, Y)$ stands for the space of $Y$-valued functions of class $\mathcal{C}^{m}$.

$\mathcal{C}_{0}^{m}(U, Y)=\left\{u \in \mathcal{C}^{m}(U, Y)\right.$; spt $u$ is compact $\}$.

$L^{p}(U), 1 \leqq p \leqq \infty$, denotes the space of $p$-summable functions with respect to the $k$-dimensional Lebesgue measure $L_{k}$.

$W^{m, p}(\Omega)=\left\{u \in L^{p}(\Omega): D^{\alpha} u \in L^{p}(\Omega)\right.$ for $\left.|\alpha| \leqq m\right\}$.

$W_{0}^{m, p}(\Omega)=$ the closure of $\mathcal{C}_{0}^{\infty}(\Omega)$ in $W^{m, p}(\Omega)$. 
Acknowledgement: 1) The authors would like to express their sincere gratitude to Professors Atsushi Inoue, Takaaki Nishida and Yoshihiro Shibata for fruitful discussions. Professor Inoue directed author's attention to the RitzGalerkin method. Without his aid $\S 3$ would have been completely different from the present form. Thanks to Professor Nishida's criticism about the errorneous statement of the energy equality in the first report [7], we can correct the error of [7].

2) Main results of the paper have already been announced briefly in [7].

\section{$\S 2$. A weak solution.}

We shall denote by $B V(\Omega)$ the space of all functions of bounded variation in the domain $\Omega \subset \boldsymbol{R}^{n}$, i. e., $u \in B V(\Omega)$ if and only if $u \in L^{1}(\Omega)$ and its gradient $D u=\left(D_{1} u, D_{2} u, \cdots, D_{n} u\right)$ in the sense of distributions is an $\boldsymbol{R}^{n}$-valued Radon measure. (See Giusti [8] for the detailed theory.) We denote its total variation measure by $|D u|$. Let $U$ be an open subset of $\Omega$. Then $|D u|(U)$ is defined by the equality

$$
|D u|(U)=\sup \left|\int_{\Omega} u \operatorname{div} \phi(x) d x\right|,
$$

where $\psi(x)=\left(\psi_{1}(x), \cdots, \psi_{n}(x)\right) \in \mathcal{C}_{0}^{1}\left(U ; \boldsymbol{R}^{n}\right)$ satisfies $|\psi(x)| \leqq 1$ for each $x$. Similarly we can define the measure $(1+|D u|)^{1 / 2}$ by the following equality:

$$
(1+|D u|)^{1 / 2}(U)=\sup \left|\int_{\Omega}\left\{\psi_{0}(x)+u \operatorname{div} \phi(x)\right\} d x\right|,
$$

where $\phi(x) \in \mathcal{C}_{0}^{1}\left(U ; \boldsymbol{R}^{n}\right)$ and $\phi_{0}(x) \in \mathcal{C}_{0}^{1}(U)$ such that

$$
\psi_{0}(x)^{2}+|\psi(x)|^{2} \leqq 1 \quad \text { for each } \quad x \in U .
$$

If $u \in \mathcal{C}^{1}(\Omega)$, then

$$
\int_{\Omega}|D u|=\int_{\Omega}|D u(x)| d x, \text { and } \int_{\Omega}(1+|D u|)^{1 / 2}=\int_{\Omega}(1+|D u(x)|)^{1 / 2} d x .
$$

The latter equals the area of the hypersurface $y=u(x)$, the graph of $u(x)$, in the space $\Omega \times \boldsymbol{R}$. If $u(x) \in B V(\Omega)$, then we can define its boundary value (the trace of $u) \gamma u$ to $\partial \Omega$. $\gamma u$ belongs to $L^{1}(\partial \Omega)$. Let $g \in C^{1}\left(\boldsymbol{R}^{n} ; \boldsymbol{R}^{n}\right)$. Then we have the Green-Stokes formula

$$
\int_{\Omega} u \operatorname{div} g d x=-\int_{\Omega} D u \cdot g+\int_{\partial \Omega} \gamma u g \cdot \vec{n} d \mathscr{H}_{n-1},
$$

where $\vec{n}$ is the unit outer normal to $\partial \Omega$.

If $u \in B V(\Omega)$, then $E=\{(x, y) \in \Omega \times \boldsymbol{R}: u(x)>y\}$ is the subgraph of $u$. The characteristic function $\chi_{E}(x, y)$ of $E$ is a function of bounded variation on every bounded open subset of $\Omega \times \boldsymbol{R}$. $D \chi_{E}$ is an $\boldsymbol{R}^{n+1}$-valued Radon measure on $\Omega \times \boldsymbol{R}$. We know that spt $\left|D \chi_{E}\right| \subset \partial E$. 
For $\rho>0$, we set $B(x, y ; \rho)=\left\{(z, w) \in \boldsymbol{R}^{n} \times \boldsymbol{R}:|z-x|^{2}+|w-y|^{2}<\rho^{2}\right\}$. Then the reduced boundary $\partial^{*} E$ of $E$ is the set of all points $(x, y) \in \Omega \times \boldsymbol{R}$ with the following properties:

(i) $\int_{B(x, y \cdot \rho)}\left|D \chi_{E}\right|>0$ for each $\rho$.

(ii) The limit $\nu(x, y)=\lim _{\rho \rightarrow 0} \nu_{\rho}(x, y)$ exists, where

$$
\nu_{\rho}(x, y)=\frac{\int_{B(x, y \rho)} D \chi_{E}}{\int_{B(x, y \quad \rho)}\left|D \chi_{E}\right|}
$$

and

$$
|\nu(x, y)|=1 .
$$
$\Omega \times \boldsymbol{R}$

It is known that $\left|D \chi_{E}\right|\left(\Omega \times \boldsymbol{R} \backslash \partial^{*} E\right)=0$ and that for each Borel subset $A$ of

$$
\begin{gathered}
\left|D \chi_{E}\right|(A)=\mathscr{H}_{n}\left(A \cap \partial^{*} E\right), \\
D \chi_{E}=\nu\left|D \chi_{E}\right| .
\end{gathered}
$$

The vector $\nu(x, y)$ is considered to be the unit inner normal at $(x, y) \in \partial^{*} E$ to $\partial^{*} E$ in a generalized sense. In fact, if $u \in \mathcal{C}^{1}(\Omega)$ then $\operatorname{spt}\left|D \chi_{E}\right|=$ the graph of $u$, and

$$
\begin{aligned}
& \nu_{j}(x, u(x))=D_{j} u(x)\left(1+|D u(x)|^{2}\right)^{-1 / 2}, \quad \jmath=1,2, \cdots, n, \\
& \nu_{n+1}(x, u(x))=-\left(1+|D u(x)|^{2}\right)^{-1 / 2} .
\end{aligned}
$$

If a function $u(t, x)$ is of bounded variation with respect to $x \in \Omega$ for each fixed $t$, then the subgraph of $u(t, *)$ will be denoted by $E(t)$. Notations $D \chi_{E(t)}$, and $\nu(t ; x, y)$ etc. have obvious meanings.

DeFinition 2.1. Let $\omega$ be an open subset of $\Omega$ and $(a, b)$ be a time interval. Then a function $u(t, x) \in L_{\text {loc }}^{1}((a, b) \times \omega)$ is said to be a $B V$-solution of the equation (1.1) in $(a, b) \times \omega$ if $u(t, x)$ is a function of bounded variation with respect to $x \in \omega$ for any fixed $t \in(a, b)$ and it satisfies the equation

$$
\begin{array}{r}
\int_{a}^{b} d t \int_{\omega \times \boldsymbol{R}}\left\{D_{t}^{2} \psi(t, x) u(t, x)+\sum_{\jmath=1}^{n} D_{\jmath} \psi(t, x) \nu_{j}(t ; x, y)\right\} \\
\times \nu_{n+1}(t ; x, y)\left|D \chi_{E(t)}\right|=0
\end{array}
$$

for any function $\phi(t, x) \in \mathcal{C}_{0}^{\infty}((a, b) \times \omega)$.

As to the initial-boundary value problem (1.1), (1.2) and (1.3) we use the following definition.

Definition 2.2. Assume that $u_{0} \in B V(\Omega)$ and $u_{1} \in L^{2}(\Omega)$. Let $T>0$ be any 
number. Then a function $u(t, x) \in L_{\text {loc }}^{1}(\boldsymbol{R} \times \Omega)$ is called a $B V$-solution of the equations (1.1), (1.2) and (1.3) for $0 \leqq t<T$ if the following conditions hold:

(i) For each $t \in \boldsymbol{R}, u(t, x)$ is a function of bounded variation with respect to $x$ such that $\gamma u=0$.

(ii) For each $\phi(t, x) \in \mathcal{C}^{2}\left([0, T) ; \mathcal{C}_{0}(\Omega)\right) \cap \mathcal{C}\left([0, T) ; \mathcal{C}^{2}(\Omega)\right)$ vanishing near $t=T$, we have

$$
\begin{aligned}
& \int_{0}^{T} d t \int_{\Omega \times \boldsymbol{R}}\left\{D_{t}^{2} \psi(t, x) u(t, x)+\sum_{j=1}^{n} D_{j} \psi(t, x) \nu_{j}(t ; x, y)\right\} \nu_{n+1}(t ; x, y)\left|D \chi_{E(t)}\right| \\
& \quad=-\int_{\Omega} \psi(0, x) u_{1}(x) d x+\int_{\Omega} D_{t} \psi(0, x) u_{0}(x) d x .
\end{aligned}
$$

If $u(t, x) \in C^{1}([0, T) \times \Omega)$, then the above definition coincides with the usual definition of a weak solution.

\section{$\S 3$. Definition of a varifold solution.}

Let $G=G(n+1, n)$ be the Grassmann manifold of all $n$-dimensional vector subspaces of $\boldsymbol{R}^{n+1}$. Let $S \in G$ be an $n$-dimensional vector subspace in $\boldsymbol{R}^{n+1}$. Then we denote the unit normal to $S$ by $\nu(S)=\left(\nu_{1}(S), \cdots, \nu_{n+1}(S)\right)$. We choose $\nu(S)$ so that $\nu_{n+1}(S) \leqq 0$. If $\nu_{n+1}(S)=0$, then $\nu(S)$ is not unique. We call the set $\operatorname{irr}(G)=\left\{S \in G: \nu_{n+1}(S)=0\right\}$ the set of irregularity. Functions $\nu_{n+1}(S)$ and $\nu_{n+1}(S) \nu_{j}(S), j=1,2, \cdots, n$, are single-valued continuous functions on $G$. A point of $\Omega \times \boldsymbol{R} \times G$ is denoted by $(x, y, S)$.

A varifold (an $n$-varifold, more precisely), $V(x, y, S)$ is a positive Radon measure on $\Omega \times \boldsymbol{R} \times G$. (See Allard [1] for detailed discussions).

Example 3.1. If $u \in B V(\Omega)$, then $u$ (or the graph of $u$, more precisely) is identified with a varifold $V(x, y, S)$ in the following manner: For any $\phi(x, y, S) \in \mathcal{C}_{0}(\Omega \times \boldsymbol{R} \times G)$,

$$
\int_{\Omega \times R \times G} \psi(x, y, S) d V(x, y, S)=\int_{\partial * E} \psi\left(x, y, \operatorname{Tan}_{(x, y)}\left(\partial^{*} E\right)\right)\left|D \chi_{E}\right|,
$$

where $\operatorname{Tan}_{(x, y)} \partial^{*} E$ is the tangent hyperplane at $(x, y)$ to the reduced boundary $\partial^{*} E$. We call this identification canonical.

Keeping this example in mind, we can introduce the following

Definition 3.1. Let $\omega$ be an open subset of $\Omega$. A varifold $V(t ; x, y, S)$ depending on a parameter $t \in(a, b)$ is called a varifold solution of the equation (1.1) for $(a, b) \times \omega \subset \boldsymbol{R} \times \Omega$ if and only if the following two conditions hold:

$$
\int_{a}^{b} d t \int_{\omega \times G} d V(t ; x, y, S)<\infty .
$$

And the equality 


$$
\begin{aligned}
0= & \int_{a}^{b} d t \int_{\omega \times \boldsymbol{R} \times G} D_{t}^{2} \psi(t, x) y \nu_{n+1}(S) d V(t ; x, y, S) \\
& +\int_{a}^{b} d t \int_{\omega \times \boldsymbol{R} \times G}\left\{\sum_{j=1}^{n} D_{j} \psi(t, x) \nu_{\jmath}(S) \nu_{n+1}(S)\right\} d V(t ; x, y, S)
\end{aligned}
$$

holds for any function $\phi(t, x)$ in $\mathcal{C}_{0}^{\infty}((a, b) \times \omega)$.

Corresponding to Definition 2.2 we introduce the following

Definition 3.3. Let $T$ be a positive number. A varifold $V(t ; x, y, S)$ depending on a parameter $t \in \boldsymbol{R}$ is called a varifold solution of the equation (1.1) and (1.2) for $[0, T)$ if and only if the following two conditions hold:

$$
\int_{0}^{T} d t \int_{\omega \times R \times G} d V(t ; x, y, S)<\infty .
$$

And the equality

$$
\begin{aligned}
& \int_{0}^{T} d t \int_{\Omega \times \boldsymbol{R} \times G} D_{t}^{2} \psi(t, x) y \nu_{n+1}(S) d V(t ; x, y, S) \\
& \quad+\int_{0}^{T} d t \int_{\Omega \times \boldsymbol{R} \times G}\left\{\sum_{j=1}^{n} D_{j} \psi(t, x) \nu_{j}(S) \nu_{n+1}(S)\right\} d V(t ; x, y, S) \\
& \quad=-\int_{\Omega} \psi(0, x) u_{1}(x) d x+\int_{\Omega} D_{t} \psi(0, x) u_{0}(x) d x
\end{aligned}
$$

holds for any function $\phi(t, x)$ in $\mathcal{C}^{2}\left([0, T) ; \mathcal{C}_{0}(\Omega)\right) \cap \mathcal{C}\left([0, T) ; \mathcal{C}^{2}(\Omega)\right)$ vanishing near $t=T$.

If a varifold solution $V(t ; x, y, S)$ can be canonically identified with a function $u(t, x)$ of bounded variation as in Example 3.1, then $u(t, x)$ is a $B V$ solution of (1.1) and (1.3). This is because

$$
\begin{aligned}
& \int_{\Omega \times R \times G} D_{t}^{2} \psi(t, x) y \nu_{n+1}(S) d V(t ; x, y, S) \\
= & \int_{\Omega \times R} D_{t}^{2} \psi(t, x) u(t, x) \nu_{n+1}(t ; x, y)\left|D \chi_{E(t)}\right|,
\end{aligned}
$$

and

$$
\begin{aligned}
& \int_{\Omega \times \boldsymbol{R} \times G} D_{\jmath} \phi(t, x) \boldsymbol{\nu}_{\jmath}(S) \nu_{n+1}(S) d V(t ; x, y, S) \\
= & \int_{\Omega \times \boldsymbol{R}} D_{j} \phi(t, x) \nu_{\jmath}(t ; x, y) \nu_{n+1}(t ; x, y)\left|D \chi_{E(t)}\right| .
\end{aligned}
$$

\section{§4. Existence of a global varifold solution.}

Now we state the main theorem. 
THEOREM 1. Assume that $u_{0} \in W_{0}^{1,2}(\Omega)$ and $u_{1} \in L^{2}(\Omega)$. Then there exists a varifold solution $V(t ; x, y, S)$ of $(1.1)$ and $(1.2)$, that $\imath s, V(t ; x, y, S)$ satisfies (3.2) and (3.3) for any $T>0$. section.

Proof is done by the Ritz-Galerkin method, which occupies the rest of this

Let $\phi_{k}(x), k=1,2, \cdots$, be the normalized eigen-functions of the Dirichlet problem in $\Omega$ :

$$
\begin{gathered}
-\Delta \phi_{k}(x)=\lambda_{k} \psi_{k}(x), \quad x \in \Omega, \\
\phi_{k}(x)=0 \quad \text { if } \quad x \in \partial \Omega .
\end{gathered}
$$

The system $\left\{\psi_{k}\right\}_{k=1}^{\infty}$ forms a complete ortho-normal system in $L^{2}(\Omega)$. For $m=$ $1,2, \cdots$, we put

$$
P_{m} f(x)=\sum_{k=1}^{m}\left(f, \phi_{k}\right) \psi_{k}(x) .
$$

The $m$-th approximate solution of (1.1) is of the form

$$
u^{m}(t, x)=\sum_{k=1}^{m} a_{k}^{m}(t) \phi_{k}(x)
$$

and satisfies the equation

$$
\begin{gathered}
P_{m}\left\{D_{t}^{2} u^{m}(t, x)-\sum_{\jmath=1}^{n} D_{j}\left(D_{\jmath} u^{m}(t, x)\left(1+\left|D u^{m}(t, x)\right|^{2}\right)^{-1 / 2}\right)\right\}=0, \\
u^{m}(0, x)=P_{m} u_{0}, \quad D_{t} u^{m}(0, x)=P_{m} u_{1} .
\end{gathered}
$$

This is equivalent to the system of equations

$$
\begin{gathered}
D_{t}^{2} a_{k}^{m}(t)+\sum_{j=1}^{n} \int_{\Omega} D_{j} \psi_{k}(x)\left\{D_{j} u^{m}(t, x)\left(1+\left|D u^{m}(t, x)\right|^{2}\right)^{-1 / 2}\right\} d x=0, \\
a_{k}^{m}(0, x)=\left(u_{0}, \phi_{k}\right), \quad D_{t} a_{k}^{m}(0, x)=\left(u_{1}, \phi_{k}\right),
\end{gathered}
$$

for $k=1,2, \cdots, m$.

Proposition 4.1. The m-th approxımate solution $u^{m}(t, x)$ exists for all $t \in \boldsymbol{R}$.

Proof. Let $A_{m}(t)=\left(a_{1}^{m}(t), a_{2}^{m}(t), \cdots, a_{m}^{m}(t)\right)$. Then the correspondence

$$
A_{m}(t) \rightarrow F_{j k}\left(A^{m}\right)=\int_{\Omega} D_{j} \psi_{k}(x) D_{\jmath} u^{m}(t, x)\left(1+\left|D u^{m}(t, x)\right|^{2}\right)^{-1 / 2} d x
$$

is uniformly Lipschitz continuous for $k=1,2, \cdots, m$, and $j=1,2, \cdots, n$. This proves Proposition.

Proposition 4.2. (Energy estimate). For $m=1,2, \cdots$, 


$$
\begin{aligned}
& \frac{1}{2} \int_{\Omega}\left|D_{t} u^{m}(t, x)\right|^{2} d x+\int_{\Omega}\left(1+\left|D u^{m}(t, x)\right|^{2}\right)^{1 / 2} d x \\
& \quad=\frac{1}{2} \int_{\Omega}\left|P_{m} u_{1}(x)\right|^{2} d x+\int_{\Omega}\left(1+\left|D P_{m} u_{0}(x)\right|^{2}\right)^{1 / 2} d x
\end{aligned}
$$

In particular,

$$
\frac{1}{2} \int_{\Omega}\left|D_{t} u^{m}(t, x)\right|^{2} d x+\int_{\Omega}\left(1+\left|D u^{m}(t, x)\right|^{2}\right)^{1 / 2} d x \leqq M
$$

where

$$
M=\frac{1}{2} \int_{\Omega}\left|u_{1}(x)\right|^{2} d x+\left(|\Omega|+\left\|u_{0}\right\|_{W^{1,2}(\Omega)}\right)^{1 / 2}|\Omega|^{1 / 2}
$$

Proof. Multiply both sides of (4.3) by $D_{t} u^{m}(t, x)$ and integrate with respect to $x$. Then

$$
D_{t}\left\{\frac{1}{2} \int_{\Omega}\left|D_{t} u^{m}(t, x)\right|^{2} d x+\int_{\Omega}\left(1+\left|D u^{m}(t, x)\right|^{2}\right)^{1 / 2} d x\right\}=0 .
$$

This and the initial condition (4.4) give (4.7).

To prove (4.8) we note that $\int\left|P_{m} u_{1}(x)\right|^{2} d x \leqq \int\left|u_{1}(x)\right|^{2} d x$ and that

$$
\int_{\Omega}\left(1+\left|D P_{m} u_{0}(x)\right|^{2}\right)^{1 / 2} d x \leqq\left\{\int_{\Omega}\left(1+\left|D P_{m} u_{0}(x)\right|^{2}\right) d x\right\}^{1 / 2}|\Omega|^{1 / 2} .
$$

Since $\phi_{k}$ satisfies (4.1), we have

$$
\begin{aligned}
\int_{\Omega}\left|D P_{m} u_{0}(x)\right|^{2} d x & =\left(-\Delta P_{m} u_{0}, u_{0}\right)=\sum_{j=1}^{m} \lambda_{k}\left(u_{0}, \phi_{k}\right)^{2} \\
& \leqq \sum_{j=1}^{\infty} \lambda_{j}\left(u_{0}, \phi_{k}\right)^{2}=-\left(\Delta u_{0}, u_{0}\right)=\int_{\Omega}\left|D u_{0}\right|^{2} d x .
\end{aligned}
$$

This proves (4.8) and (4.9).

For each $m=1,2,3, \cdots$, the function $u^{m}(t, x)$ is of class $\mathcal{C}^{\infty}$. We identify this with a varifold $V^{m}(t ; x, y, S)$ as in the Example 3.1 of $\S 3$. We rewrite (4.3) and (4.4) in terms of $V^{m}(t ; x, y, S)$. Let $\phi(t) \in \mathcal{C}^{2}(\boldsymbol{R})$ vanishing near $t=T$. Then we multiply both sides of (4.3) by $\phi(t) \phi_{k}(x), k \leqq m$. After integration by parts we have

$$
\begin{aligned}
\phi(0) & \int_{\Omega} u_{1}(x) \psi_{k}(x) d x-D_{t} \phi(0) \int_{\Omega} u_{0}(x) \psi_{k}(x) d x \\
= & \int_{0}^{T} d t D_{t}^{2} \phi(t) \int_{\Omega} \psi_{k}(x) u^{m}(t, x) d x \\
& +\int_{0}^{T} d t \phi(t) \int_{\Omega} \sum_{j=1}^{n} D_{\jmath} \psi_{k}(x) D_{j} u^{m}(t, x)\left(1+\left|D u^{m}(t, x)\right|^{2}\right)^{-1 / 2} d x
\end{aligned}
$$


On the other hand, we have, by definition,

$$
\int_{\Omega} \phi_{k}(x) u^{m}(t, x) d x=-\int_{\Omega \times R \times G} \phi_{k}(x) y \nu_{n+1}(S) d V^{m}(t ; x, y, S)
$$

and

$$
\begin{aligned}
& \int_{\Omega} D_{J} \phi_{k}(x)\left\{D_{j} u^{m}(t, x)\left(1+\left|D u^{m}(t, x)\right|^{2}\right)^{-1 / 2}\right\} d x \\
& =-\int_{\Omega \times R \times G} D_{j} \psi_{k}(x) \boldsymbol{\nu}_{j}(S) \boldsymbol{\nu}_{n+1}(S) d V^{m}(t ; x, y, S) .
\end{aligned}
$$

Therefore $V^{m}(t ; x, y, S)$ satisfies the following equation :

$$
\begin{aligned}
& \int_{0}^{T} D_{t}^{2} \phi(t) d t \int_{\Omega \times R \times G} \phi_{k}(x) y \nu_{n+1}(S) d V^{m}(t ; x, y, S) \\
& \quad+\int_{0}^{T} \phi(t) d t \int_{\Omega \times R \times G}\left\{\sum_{j=1}^{n} D_{j} \psi_{k}(x) \nu_{j}(S) \nu_{n+1}(S)\right\} d V^{m}(t ; x, y, S) \\
& =-\int_{\Omega} \phi(0) u_{1}(x) \phi_{k}(x) d x+\int_{\Omega} D_{t} \phi(0) u_{0}(x) \phi_{k}(x) d x
\end{aligned}
$$

where $k=1,2, \cdots, m$ and $\phi(t)$ is an arbitrary function in $\mathcal{C}_{0}^{2}(\boldsymbol{R})$ vanishing near $t=T$.

We wish to choose a subsequence $\left\{m^{\prime}\right\} \subset\{m\}$ so that $\lim _{m^{\prime} \rightarrow \infty} V^{m^{\prime}}(t ; x, y, S)$ exists. In fact we have

Proposition 4.3. There exist a subset $\boldsymbol{R}_{1}$ of $\boldsymbol{R}$, subsequence $\left\{m^{\prime}\right\}$ of $\{m\}$ and a varifold $V(t ; x, y, S)$ depending on a parameter $t \in \boldsymbol{R}_{1}$ with the following properties: $L_{1}\left(\boldsymbol{R} \backslash \boldsymbol{R}_{1}\right)=0$ and

$$
\begin{aligned}
& \int_{-\infty}^{\infty} \phi(t) d t \int_{\Omega \times R \times G} \xi(x, y, S) d V(t ; x, y, S) \\
& \quad=\lim _{m^{\prime} \rightarrow \infty} \int_{-\infty}^{\infty} \phi(t) d t \int_{\Omega \times R \times G} \xi(x, y, S) d V^{m^{\prime}}(t ; x, y, S),
\end{aligned}
$$

for any $\phi(t) \in L^{1}(\boldsymbol{R})$ and $\xi(x, y, S) \in \mathcal{C}_{0}(\Omega \times \boldsymbol{R} \times G)$. We have

$$
\int_{\Omega \times \boldsymbol{R} \times G} d V(t ; x, y, S) \leqq M .
$$

Proof. Let $M$ be the constant in (4.9). Then we note that

$$
\begin{aligned}
\int_{\Omega \times \boldsymbol{R} \times G} d V^{m}(t ; x, y, S) & =\int_{\left(y=u^{m}(t, x)\right)} d \mathscr{H}_{n} \\
& =\int_{\Omega}\left(1+\left|D u^{m}(t, x)\right|^{2}\right)^{1 / 2} d x \\
& \leqq M .
\end{aligned}
$$


If $\xi(x, y, S) \in \mathcal{C}_{0}(\Omega \times \boldsymbol{R} \times G)$ then

$$
\left\langle\xi, V^{m}(t)\right\rangle=\int_{\Omega \times \boldsymbol{R} \times G} \xi(x, y, S) d V^{m}(t ; x, y, S)
$$

is a bounded function of $t$, because we have the estimate

$$
\left|\left\langle\xi, V^{m}(t)\right\rangle\right| \leqq M \max |\xi(x, y, S)| .
$$

We consider the family of mappings $\mathcal{C}_{0}(\Omega \times \boldsymbol{R} \times G) \ni \xi \rightarrow\left\langle\xi, V^{m}(t)\right\rangle \in L^{\infty}(\boldsymbol{R})$. The estimate (4.14) implies that this family of mappings is equicontinuous and that for each $\xi$ the image of mappings is relatively compact in the weak* topology of $L^{\infty}(\boldsymbol{R})$. We can apply the Ascoli-Arzela theorem because $\mathcal{C}_{0}(\Omega \times \boldsymbol{R} \times G)$ is separable. And there exists a subsequence $\left\{V^{m^{\prime}}(t ; x, y, S)\right\}_{m^{\prime}}$ such that

$$
w^{*}-\lim _{m^{\prime} \rightarrow \infty}\left\langle\xi, V^{m^{\prime}}(t)\right\rangle=f(t ; \xi)
$$

exists in $L^{\infty}(\boldsymbol{R})$ for each $\xi$. It is clear that $f(t ; \xi) \geqq 0$ if $\xi \geqq 0$. And we have

$$
\|f(t ; \xi)\|_{L^{\infty}} \leqq M \max |\xi(x, y, S)| .
$$

The function $f(t ; \xi)$ may not be defined for $t$ in an exceptional set of $L_{1}$-measure 0 and this exceptional set may depend on $\xi$. To avoid this inconvenience we choose a good representative $V(t ; \xi)$ of $f(t ; \xi)$ as a function of $t$ : We define

$$
V(t ; \xi)=\lim _{h \rightarrow+0} \frac{1}{2 h} \int_{t-h}^{t+h} f(t ; \xi) d t .
$$

This exists and is equal to $f(t ; \xi)$ at $L_{1}$-almost every $t \in \boldsymbol{R}$ if $\xi$ is fixed. Let $\left\{\xi_{k}\right\}_{k=1}^{\infty}$ be a countable dense subset of $\mathcal{C}_{0}(\Omega \times \boldsymbol{R} \times G)$. Then the set

$$
\boldsymbol{R}_{1}=\left\{t \in \boldsymbol{R}: V\left(t ; \boldsymbol{\xi}_{k}\right) \text { exists and is finite for all } k\right\}
$$

is measurable and $L_{1}\left(\boldsymbol{R} \backslash \boldsymbol{R}_{1}\right)=0$.

We claim that $V(t ; \xi)$ exists for all $\xi \in \mathcal{C}_{0}(\Omega \times \boldsymbol{R} \times G)$ and for $t \in \boldsymbol{R}_{1}$. In fact, for any $\varepsilon>0$, there exists a function $\xi_{k}$ such that

$$
\left|\xi(x, y, S)-\xi_{k}(x, y, S)\right|<\varepsilon \quad \text { for any }(x, y, S) \in \Omega \times \boldsymbol{R} \times G .
$$

Then we have for each $t \in \boldsymbol{R}_{1}$

$$
\begin{aligned}
& \frac{1}{2 h}\left|\int_{t-h}^{t+h} f(t ; \xi) d t-\int_{t-h}^{t+h} f\left(t ; \xi_{k}\right) d t\right| \\
& \quad \leqq \frac{1}{2 h} \int_{t-h}^{t+h}\left|f\left(t ; \xi-\xi_{k}\right)\right| d t \\
& \quad \leqq M \varepsilon .
\end{aligned}
$$

The last estimate follows from (4.16) and (4.18). Hence 


$$
\begin{aligned}
V\left(t ; \xi_{k}\right)-\varepsilon & \leqq \liminf _{h \rightarrow+0} \frac{1}{2 h} \int_{t-h}^{t+h} f(t ; \xi) d t \\
& \leqq \limsup _{h \rightarrow+0} \frac{1}{2 h} \int_{t-h}^{t+h} f(t ; \xi) d t \leqq V\left(t ; \xi_{k}\right)+\varepsilon
\end{aligned}
$$

Since $\varepsilon$ is arbitrary,

$$
\lim _{h \rightarrow+0} \frac{1}{2 h} \int_{t-h}^{t+h} f(t ; \xi) d t=V(t ; \xi)
$$

exists at every $t \in \boldsymbol{R}_{1}$.

If $t \in \boldsymbol{R}_{1}$, then it follows from (4.16) and (4.17) that

$$
|V(t ; \xi)| \leqq M \max |\xi(x, y, S)| \text {. }
$$

This implies that the correspondence $\xi \rightarrow V(t ; \xi), t \in \boldsymbol{R}_{1}$, defines a Radon measure $V(t ; x, y, S)$ such that

$$
V(t ; \xi)=\int_{\Omega \times R \times G} \xi(x, y, S) d V(t ; x, y, S) .
$$

We know that $V(t ; \xi) \geqq 0$ if $\xi \geqq 0$. Therefore $V(t ; x, y, S)$ is a varifold. Clearly we have

$$
\int_{\Omega \times R \times G} d V(t ; x, y, S) \leqq M .
$$

Equality (4.15) leads us to the equality

$$
w^{*}-\lim _{m^{\prime} \rightarrow \infty}\left\langle\xi, V^{m^{\prime}}(t)\right\rangle=\langle\xi, V(t)\rangle
$$

as an element of $L^{\infty}(\boldsymbol{R})$. This proves Proposition.

End of the Proof of Theorem 1. We complete the proof of Theorem 1 by showing that the varifold $V(t ; x, y, S)$ satisfies the equality (3.3). We choose the subsequence $\left\{m^{\prime}\right\}$ as in Proposition 4.3 and denote it as $\{m\}$ in the following for the sake of brevity. Take $\phi(t) \in \mathcal{C}^{2}(\boldsymbol{R})$ which vanishes near $t=T$. Then $D_{t}^{2} \phi(t) \in L^{1}(\boldsymbol{R})$. On the other hand, we know that $\phi_{j}(x) y \nu_{n+1}(S) \in C_{0}(\Omega \times \boldsymbol{R} \times G)$. Therefore the above Proposition 4.3 asserts that

$$
\begin{gathered}
\lim _{m \rightarrow \infty} \int_{0}^{T} D_{t}^{2} \phi(t) d t \int_{\Omega \times R \times G} \phi_{k}(x) y \nu_{n+1}(S) d V^{m}(t ; x, y, S) \\
\quad=\int_{0}^{T} D_{t}^{2} \phi(t) d t \int_{\Omega \times R \times G} \psi_{k}(x) y \nu_{n+1}(S) d V(t ; x, y, S) .
\end{gathered}
$$

Similarly we have

$$
\begin{gathered}
\lim _{m \rightarrow \infty} \int_{0}^{T} \phi(t) d t \int_{\Omega \times \boldsymbol{R} \times G} \sum_{j=1}^{n} D_{j} \phi_{k}(x) \boldsymbol{\nu}_{j}(S) \boldsymbol{\nu}_{n+1}(S) d V^{m}(t ; x, y, S) \\
\quad=\int_{0}^{T} \phi(t) d t \int_{\Omega \times \boldsymbol{R} \times G} \sum_{j=1}^{n} D_{\jmath} \phi_{k}(x) \boldsymbol{\nu}_{j}(S) \boldsymbol{\nu}_{n+1}(S) d V(t ; x, y, S),
\end{gathered}
$$


because $\phi \in L^{1}(\boldsymbol{R})$ and $D_{j} \psi_{k}(x)_{j}(S) \nu_{n+1}(S) \in \mathcal{C}_{0}(\Omega \times \boldsymbol{R} \times G)$. Letting $m$ go to $\infty$ in (4.11), and using (4.21) and (4.22), we have

$$
\begin{aligned}
& \int_{0}^{T} D_{t}^{2} \phi(t) d t \int_{\Omega \times R \times G} \phi_{k}(x) y \nu_{n+1}(S) d V(t ; x, y, S) \\
& \quad+\int_{0}^{T} \phi(t) d t \int_{\Omega \times R \times G} \sum_{\jmath=1}^{n} D_{\jmath} \psi_{k}(x) \nu_{j}(S) \nu_{n+1}(S) d V(t ; x, y, S) \\
& \quad=-\int_{\Omega} \phi(0) u_{1}(x) \psi_{k}(x) d x+\int_{\Omega} D_{t} \phi(0) u_{0}(x) \psi_{k}(x) d x
\end{aligned}
$$

for $k=1,2, \cdots$. Since functions of the form $\phi(t) \phi_{k}(x)$ are total in the space $\mathcal{C}^{2}\left([0, T) ; \mathcal{C}_{0}(\Omega)\right) \cap \mathcal{C}\left([0, T) ; \mathcal{C}^{2}(\Omega)\right)$, the equality (3.5) follows from (4.23). Inequality (3.4) is a consequence of (4.13). This proves our theorem.

\section{$\S 5$. Convergence of $u^{m}(t, x)$ in the $B V$-space.}

As we have proved the global existence of a varifold solution, we wish to identify $V(t ; x, y, S)$ with a graph of a function. A graph of a function is, measure theoretically, a special case of an $n$-rectifiable subset of $\Omega \times \boldsymbol{R}$. Thus we can state our problem in the following form:

(Q) Can one identify the varifold solution $V(t ; x, y, S)$ of the preceding section with an $H_{n}$ rectifiable subset of $\Omega \times \boldsymbol{R}$ for all $t$ ?

Unfortunately we did not succeed in giving answer to this fundamental question. Of course the most probable candidate of the $H_{n}$-rectifiable subset of $\boldsymbol{\Omega} \times \boldsymbol{R}$ is the graph of the function $u(t, x)=\lim _{m \rightarrow \infty} u^{m}(t, x)$ if the limit exists. In the present section, we prove that $u(t, x)=\lim _{m \rightarrow \infty} u^{m}(t, x)$ actually exists in the space $B V(\Omega)$. We shall discuss the relationship of $V(t ; x, y, S)$ and $u(t, x)$ in the next section.

In the following we choose the subsequence $\left\{m^{\prime}\right\}$ as in Proposition 4.3 and denote it by $\{m\}$ for the sake of brevity. For any fixed $t \in \boldsymbol{R}$ the sequence $\left\{u^{m}(t, x)\right\}$ of $B V$-functions are bounded because of Proposition 4.2.

Proposition 5.1. There exists a subsequence $\left\{m^{\prime \prime}\right\} \subset\{m\}$ such that $\left\{u^{m^{\prime \prime}}(t, x)\right\}$ converges strongly, for any fixed $t$, to a function $u(t, x)$ in $L^{p}(\Omega), 1 \leqq p<\frac{n}{n-1}$, and that $\left\{D u^{m^{\prime \prime}}(t, x)\right\}$ converges to $D u(t, x)$ with respect to the $w^{*}$-topology of measures. $u(t, *) \in B V(\Omega)$ for fixed $t \in \boldsymbol{R}$. The function $u(t, x)$ is a Lipschitz continuous function of $t$ with values in $L^{2}(\Omega)$.

Proof. Since 


$$
u^{m}(t, x)=\int_{0}^{t} D_{s} u^{m}(s, x) d s+u_{0}(x),
$$

we have

For any $t, t^{\prime} \in \boldsymbol{R}$,

$$
\begin{aligned}
\left\|u^{m}(t, x)\right\|_{L^{2}(\Omega)} & \leqq t \sup \left\|D_{s} u^{m}(s, *)\right\|_{L^{2}(\Omega)}+\left\|u_{0}\right\|_{L^{2}(\Omega)} \\
& \leqq(2 M)^{1 / 2} t+\left\|u_{0}\right\|_{L^{2}(\Omega)} .
\end{aligned}
$$

$$
\left\|u^{m}\left(t^{\prime}, *\right)-u^{m}(t, *)\right\|_{L^{2}(\Omega)} \leqq\left\|\int_{t}^{t^{\prime}} D_{s} u^{m}(s, x) d s\right\| \leqq(2 M)^{1 / 2}\left|t^{\prime}-t\right| .
$$

Hence $t \rightarrow\left\{u^{m}(t, *)\right\} \in L^{2}(\Omega)$ is an equicontinuous family. The Ascoli-Arzela theorem enables us to choose a subsequence $\left\{u^{m^{\prime \prime}}(t, x)\right\}$ such that

$$
w-\lim _{m^{\prime \prime} \rightarrow \infty} u^{m^{\prime \prime}}(t, *)=u(t, *) \quad \text { in } \quad L^{2}(\Omega)
$$

exists for each $t \in \boldsymbol{R}$. As a consequence of this and (5.1), we have

$$
\left\|u\left(t^{\prime}, *\right)-u(t, *)\right\|_{L^{2}(\Omega)} \leqq(2 M)^{1 / 2}\left|t^{\prime}-t\right| .
$$

Therefore $u(t, *)$ is an $L^{2}(\Omega)$-valued Lipschitz continuous function.

We know from Proposition 4.2 that $\left\{u^{m^{\prime \prime}}(t, x)\right\}$ is a bounded set in $B V(\Omega)$. Since the inclusion $B V(\Omega) \subset L^{p}(\Omega), 1 \leqq p<\frac{n}{n-1}$, is a compact map, every subsequence of $\left\{u^{m^{\prime \prime}}(t, x)\right\}$ contains a subsequence which converges strongly to $u(t, *)$ in $L^{p}(\Omega)$ because $\left\{u^{m^{*}}(t, *)\right\}$ converges weakly to $u(t, *)$ in $L^{2}(\Omega)$. This implies that $\left\{u^{m^{*}}(t, x)\right\}$ converges to $u(t, *)$ strongly in $L^{p}(\Omega)$. It is clear that $u(t, *) \in B V(\Omega)$ for each $t$. For $j=1,2, \cdots, n,\left\{D_{j} u^{m^{\prime \prime}}(t, *)\right\}$ converges to $D_{j} u(t, *)$ in the sense of distribution. Therefore $\left\{D_{j} u^{m^{\prime}}(t, *)\right\}$ converges to $D_{j} u(t, *)$ in the sense of $w^{*}$-topology of measures.

Remark 5.2. We expect that $u(t, x)$ above is a $B V$-solution of the equation (1.1). However we failed in proving it. We shall prove later in Theorem 4 that $u(t, x)$ is a $B V$-solution if it satisfies the energy conservation law.

We let $E_{m}(t)$ and $\chi_{t}^{m}(x, y)$ denote the subgraph of $u^{m}(t, x)$ and its characteristic function, respectively. Similarly $E(t)$ and $\chi_{t}(x, y)$ stand for the subgraph of $u(t, x)$ and its characteristic function, respectively.

COROLlaRY 5.3. We may choose the subsequence $m^{\prime \prime}$ so that $\left\{D \chi_{t}^{m \prime}\right\}$ converges to $D \chi_{t}$ in the $w^{*}$-topology of measures.

Proof. Let $\phi(x, y) \in \mathcal{C}_{0}^{\infty}(\Omega \times \boldsymbol{R})$. Then

$$
\left|\int_{\Omega \times R}\left(\chi_{t}^{m^{\prime \prime}}(x, y)-\chi_{t}(x, y)\right) \phi(x, y) d x d y\right|
$$




$$
\begin{aligned}
& =\left|\int_{\Omega} d x \int_{u(t, x)}^{u^{m^{\prime \prime}(t, x)}} \psi(x, y) d y\right| \\
& \leqq \max |\psi(x, y)| \int_{\Omega}\left|u^{m^{\prime \prime}}(t, x)-u(t, x)\right| d x .
\end{aligned}
$$

As a consequence of this and Proposition 5.1, $\left\{\chi_{t}^{m^{n}}\right\}$ converges to $\chi_{t}$ in the sense of distribution. Hence $\left\{D \chi_{t}^{m}\right\}$ converges to $D \chi_{t}$ in the sense of distribution. This implies that $\left\{D \chi_{t}^{m *}\right\}$ converges to $D \chi_{t}$ in the $w^{*}$-topology of measures, because $\left|D \chi_{t}^{m^{*}}\right|$ are bounded.

For the sake of brevity we denote $\{m\}$ instead of $\left\{m^{\prime \prime}\right\}$.

Proposition 5.4. There exists a set $\boldsymbol{R}_{2} \subset \boldsymbol{R}$ and a function $\boldsymbol{R}_{2} \ni t \rightarrow D_{t} u\left(t,{ }^{*}\right)$ $\in L^{2}(\Omega)$ such that $L_{1}\left(\boldsymbol{R} \backslash \boldsymbol{R}_{2}\right)=0$ and

$$
\int_{\Omega} D_{t} u(t, x) \psi(x) d x=\lim _{h \rightarrow 0} h^{-1}\left\{\int_{\Omega} u(t+h, x) \psi(x) d x-\int_{\Omega} u(t, x) \psi(x) d x\right\}
$$

exists for all $\phi \in L^{2}(\Omega)$ and $t \in \boldsymbol{R}_{2}$. At $L_{1}$-almost all $t$ we have

$$
\left\|D_{t} u(t, *)\right\|_{L^{2}(\Omega)} \leqq \limsup _{m \rightarrow \infty}\left\|D_{t} u^{m}\left(t,{ }^{*}\right)\right\|_{L^{2}(\Omega)} .
$$

For any $T>0, D_{t} u(t, x)$ is the weak limet of $\left\{D_{t} u^{m}(t, x)\right\}_{m}$ in the space $L^{2}((0, T) \times \Omega)$.

Proof. For any $\phi \in L^{2}(\Omega)$, we put

$$
F(t, \phi)=\lim _{h \rightarrow 0} h^{-1}\left\{\int_{\Omega} u(t+h, x) \phi(x) d x-\int_{\Omega} u(t, x) \phi(x) d x\right\}
$$

if the right hand side exists. As a result of (5.1), we have

$$
\left|h^{-1}\left\{\int_{\Omega} u(t+h, x) \psi(x) d x-\int_{\Omega} u(t, x) \phi(x) d x\right\}\right| \leqq(2 M)^{1 / 2}\|\phi\|_{L^{2}(\Omega)} .
$$

Let $\left\{\xi_{k}(x)\right\}_{k=1}^{\infty}$ be a countable dense subset of $L^{2}(\Omega)$. Then by virtue of (5.5), we see that there exists a set $\boldsymbol{R}_{2} \subset \boldsymbol{R}$ such that $L_{1}\left(\boldsymbol{R} \backslash \boldsymbol{R}_{2}\right)=0$ and $F\left(t, \xi_{k}\right)$ exists at $t \in \boldsymbol{R}_{2}$ and $k=1,2, \cdots$, .

We claim that for any $\phi \in L^{2}(\Omega), F(t, \phi)$ exists at all $t \in \boldsymbol{R}_{2}$. In fact for given $\phi \in L^{2}(\Omega)$ and $\varepsilon>0$, there exists $\xi_{k}$ such that

$$
\left\|\xi_{k}-\psi\right\|_{L^{2}(\Omega)}<\varepsilon /(4 M)^{1 / 2} .
$$

Applying (5.5) to $\phi-\xi_{k}$, we have

$$
\begin{gathered}
h^{-1}\left\{\int_{\Omega} u(t+h, x) \xi_{k}(x) d x-\int_{\Omega} u(t, x) \xi_{k}(x) d x\right\}-\varepsilon \\
\leqq h^{-1}\left\{\int_{\Omega} u(t+h, x) \psi(x) d x-\int_{\Omega} u(t, x) \psi(x) d x\right\}
\end{gathered}
$$




$$
\leqq h^{-1}\left\{\int_{\Omega} u(t+h, x) \xi_{k}(x) d x-\int_{\Omega} u(t, x) \xi_{k}(x) d x\right\}+\varepsilon .
$$

If $t \in \boldsymbol{R}_{2}$, then

$$
\begin{aligned}
F\left(t, \xi_{k}\right)-\varepsilon & \leqq \liminf _{m \rightarrow \infty} h^{-1}\left\{\int_{\Omega} u(t+h, x) \psi(x) d x-\int_{\Omega} u(t, x) \psi(x) d x\right\} \\
& \leqq \limsup _{m \rightarrow \infty} h^{-1}\left\{\int_{\Omega} u(t+h, x) \psi(x) d x-\int_{\Omega} u(t, x) \psi(x) d x\right\} \\
& \leqq F\left(t, \xi_{k}\right)+\varepsilon .
\end{aligned}
$$

Since $\varepsilon$ is arbitrary, $F(t, \phi)$ exists.

From the estimate (5.5), we have

$$
|F(t, \phi)| \leqq(2 M)^{1 / 2}\|\phi\|_{L^{2}(\Omega)} .
$$

$F(t, \phi)$ is a continuous linear functional of $\phi \in L^{2}(\Omega)$. Therefore there exists $D_{t} u(t, *) \in L^{2}(\Omega)$ such that

$$
\int_{\Omega} D_{t} u(t, x) \psi(x) d x=F(t, \phi) .
$$

By definition we have

$$
\int_{\Omega} u(t, x) \psi(x) d x-\int_{\Omega} u_{0}(x) \psi(x) d x=\int_{0}^{t} d s \int_{\Omega} D_{s} u(s, x) \psi(x) d x .
$$

Let $v(x)=D_{t} u(t, x)$. Then

$$
\begin{aligned}
\|v\|_{L^{2}(\Omega)}^{2} & =\lim _{h \rightarrow 0} \frac{1}{2 h} \int_{t-h}^{t+h} d \tau \int_{\Omega} D_{\tau} u(\tau, x) v(x) d x \\
& =\lim _{m \rightarrow \infty} \frac{1}{2 h}\left\{\int_{\Omega} u(t+h, x) v(x) d x-\int_{\Omega} u(t-h, x) v(x) d x\right\} \\
& =\lim _{h \rightarrow 0} \lim _{m \rightarrow \infty} \frac{1}{2 h}\left\{\int_{\Omega} u^{m}(t+h, x) v(x) d x-\int_{\Omega} u^{m}(t-h, x) v(x) d x\right\} \\
& =\lim _{h \rightarrow 0} \lim _{m \rightarrow \infty} \frac{1}{2 h} \int_{t-h}^{t+h} \int_{\Omega} D_{\tau} u^{m}(\tau, x) v(x) d x d \tau \\
& \leqq \lim _{h \rightarrow 0} \frac{1}{2 h} \int_{t-h}^{t+h}\left(\limsup _{m \rightarrow \infty}\left\|D_{t} u^{m}(\tau, *)\right\|_{L^{2}(\Omega)}\|v\|_{L^{2}(\Omega)}\right) d \tau \\
& \leqq\|v\|_{L^{2}(\Omega)} \limsup _{m \rightarrow \infty}\left\|D_{t} u(t, *)\right\|_{L^{2}(\Omega)}
\end{aligned}
$$

at $L_{1}$ almost all $t$. Therefore

$$
\|v\|_{L^{2}(\Omega)} \leqq \limsup _{m \rightarrow \infty}\left\|D_{t} u^{m}(t, *)\right\|_{L^{2}(\Omega)}
$$

at $L_{1}$-almost all $t$. 
The energy inequality (4.8) implies that for any $T>0,\left\{D_{t} u^{m}(t, x)\right\}$ is bounded in $L^{2}((0, T) \times \Omega)$. Let $\left\{D_{t} u^{m^{\prime}}(t, x)\right\}$ be any weakly convergent subsequence of $\left\{D_{t} u^{m}(t, x)\right\}$ and let $w(t, x)$ be its limit. Then

$$
\int_{\Omega} u^{m^{\prime}}(t, x) \psi(x) d x-\int_{\Omega} P_{m^{\prime}} u_{0}(x) \psi(x) d x=\int_{0}^{t} d s \int_{\Omega} D_{s} u^{m^{\prime}}(s, x) \psi(x) d x .
$$

Taking the limit of this as $m^{\prime} \rightarrow \infty$, we have

$$
\int_{\Omega} u(t, x) \psi(x) d x-\int_{\Omega} u_{0}(x) \phi(x) d x=\int_{0}^{t} d s \int_{\Omega} w(s, x) \phi(x) d x .
$$

If follows from this and (5.7) that $D_{t} u(t, x)=w(t, x)$ at almost every $(t, x)$. This proves Proposition 5.4.

\section{§6. Varifold solution and $B V$ function.}

In this section we discuss the relationship of the varifold solution $V(t ; x, y, S)$ of $\S 4$ and the $B V$-function $u(t, x)$ given in $\S 5$. We prove that the varifold $V(t ; x, y, S)$ can be identified with the graph of the function $u(t, x)$ if $u(t, x)$ satisfies the energy conservation law. For the sake of brevity we denote $\left\{m^{\prime \prime}\right\}$ by $\{m\}$.

Definition 6.1. As in Allard [1], we define the weight measure $\|V(t)\|$ of the varifold $V(t ; x, y, S)$ by the equality

$$
\int_{\Omega \times \boldsymbol{R}} \psi(x, y) d\|V(t)\|=\int_{\Omega \times \boldsymbol{R} \times G} \psi(x, y) d V(t ; x, y, S)
$$

for any $\psi(x, y)$ in $\mathcal{C}_{0}(\Omega \times \boldsymbol{R})$. Similarly, for $\jmath=1,2, \cdots, n+1$, we define the measure $\| V(t)\left\llcorner\nu_{j} \|\right.$ by the equality

$$
\int_{\Omega \times \boldsymbol{R}} \psi(x, y) d \| V(t)\left\llcorner\nu_{\jmath} \|=\int_{\Omega \times \boldsymbol{R} \times G} \psi(x, y) \nu_{\jmath}(S) d V(t ; x, y, S) .\right.
$$

As in $\S 5$ we denote by $E(t)$ and $E_{m}(t)$ the subgraphs of $u(t, x)$ and $u^{m}(t, x)$, respectively. And we denote by $\chi_{t}$ and $\chi_{t}^{m}$ the characteristic functions of $E(t)$ and $E_{m}(t)$, respectively. Then

Proposition 6.2. (i) For each $\phi \in L^{1}(\boldsymbol{R})$ and for any $\phi(x, y) \in \mathcal{C}_{0}(\Omega \times \boldsymbol{R})$, we have

$$
\int_{\boldsymbol{R}} \phi(t) d t \int_{\Omega \times \boldsymbol{R}} \phi(x, y) d\|V(t)\|=\lim _{m \rightarrow \infty} \int_{\boldsymbol{R}} \phi(t) d t \int_{\Omega \times \boldsymbol{R}} \phi(x, y)\left|D \chi_{t}^{m}\right| .
$$

(ii) There exists a subset $\boldsymbol{R}_{3}$ of $\boldsymbol{R}$ with the following propertzes $\cdot L_{1}\left(\boldsymbol{R} \backslash \boldsymbol{R}_{3}\right)$ $=0$ and for any $t \in \boldsymbol{R}_{3}$ and $\phi \in \mathcal{C}_{0}(\Omega \times \boldsymbol{R})$, we have 


$$
\begin{aligned}
& \liminf _{m \rightarrow \infty} \int_{\Omega \times \boldsymbol{R}} \phi(x, y)\left|D \chi_{t}^{m}\right| \\
& \leqq \int_{\Omega \times \boldsymbol{R}} \phi(x, y) d\|V(t)\| \leqq \limsup _{m \rightarrow \infty} \int_{\Omega \times \boldsymbol{R}} \phi(x, y)\left|D \chi_{t}^{m}\right| .
\end{aligned}
$$

(iii) For any open subset $B \subset \Omega \times \boldsymbol{R}$ and any compact set $K \subset B$, we have

$$
\limsup _{m \rightarrow \infty}\left|D \chi_{t}^{m}\right|(B) \geqq\|V(t)\|(B) \geqq \liminf _{m \rightarrow \infty}\left|D \chi_{t}^{m}\right|(K)
$$

for $t \in \boldsymbol{R}_{3}$.

(iv) Assume that $B$ is a bounded open subset of $\Omega \times \boldsymbol{R}$. Assume further that for some $t \in \boldsymbol{R}_{3}$

$$
\lim _{m \rightarrow \infty}\left|D \chi_{t}^{m}\right|(B) \text { exists }
$$

and

$$
\|V(t)\|(\partial B)=0
$$

Then

$$
\|V(t)\|(B)=\lim _{m \rightarrow \infty}\left|D \chi_{t}^{m}\right|(B)
$$

Proof. (i) Using Proposition 4.3, we have

$$
\begin{aligned}
& \int_{R} \phi(t) d t \int_{\Omega \times R} \phi(x, y) d\|V(t)\| \\
& \quad=\lim _{m \rightarrow \infty} \int_{R} \phi(t) d t \int_{\Omega \times R} \psi(x, y) d V^{m}(t ; x, y, S) \\
& \quad=\lim _{m \rightarrow \infty} \int_{R} \phi(t) d t \int_{\Omega \times R} \psi(x, y)\left|D \chi_{t}^{m}\right| .
\end{aligned}
$$

This proves (i).

Proof of (ii). Let $\left\{\xi_{k}(x, y)\right\}_{k=1}^{\infty}$ be a countable dense subset of $\mathcal{C}_{0}(\Omega \times \boldsymbol{R})$. We have from Proposition 4.2 that

$$
\int \xi_{k}(x, y)\left|D \chi_{t}^{m}\right| \geqq-\max \left|\xi_{k}(x, y)\right| M .
$$

The right hand side is independent of $m$. Take $\phi \in L^{1}(\boldsymbol{R})$ so that $\phi(t) \geqq 0$. Then Fatou's lemma gives

$$
\begin{gathered}
\int_{R} \phi(t) d t\left(\liminf _{m \rightarrow \infty} \int_{\Omega \times R} \xi_{k}(x, y)\left|D \chi_{t}^{m}\right|\right) \\
\leqq \liminf _{m \rightarrow \infty} \int_{R} \phi(t) d t \int_{\Omega \times R} \xi_{k}(x, y)\left|D \chi_{t}^{m}\right|
\end{gathered}
$$




$$
\begin{aligned}
& \leqq \liminf _{m \rightarrow \infty} \int_{R} \phi(t) d t \int_{\Omega \times R} \xi_{k}(x, y) d V^{m}(t ; x, y, S) \\
& =\int_{R} \phi(t) d t \int_{\Omega \times \boldsymbol{R}} \xi_{k}(x, y) d\|V(t)\| .
\end{aligned}
$$

Similarly we can prove

$$
\int_{R} \phi(t) d t \int_{\Omega \times R} \xi_{k}(x, y) d\|V(t)\| \leqq \int_{R} \phi(t) d t \limsup _{m \rightarrow \infty} \int_{\Omega \times R} \xi_{k}(x, y)\left|D \chi_{t}^{m}\right| .
$$

As a consequence of (6.10) and (6.11), there exists a subset $\boldsymbol{R}_{3} \subset \boldsymbol{R}$ with the following properties: $L_{1}\left(\boldsymbol{R} \backslash \boldsymbol{R}_{3}\right)=0$ and we have

$$
\begin{aligned}
\liminf _{m \rightarrow \infty} \int_{\Omega \times R} \xi_{k}(x, y)\left|D \chi_{t}^{m}\right| & \leqq \int_{\Omega \times R} \xi_{k}(x, y) d\|V(t)\| \\
& \leqq \limsup _{m \rightarrow \infty} \int_{\Omega \times R} \xi_{k}(x, y)\left|D \chi_{t}^{m}\right|,
\end{aligned}
$$

for each $t \in \boldsymbol{R}_{3}$ and for all $k=1,2, \cdots$, . Since $\left\{\xi_{k}\right\}_{k}$ is dense in $\mathcal{C}_{0}(\Omega \times \boldsymbol{R})$, (6.4) holds for any $\phi \in \mathcal{C}_{0}(\Omega \times \boldsymbol{R})$ and $t \in \boldsymbol{R}_{3}$.

(iii) Let $\phi \in \mathcal{C}_{0}(B)$ be a function such that $0 \leqq \phi(x, y) \leqq 1$ and $\phi(x, y)=1$ on $K$. Let $t \in \boldsymbol{R}_{3}$. Then we have from (6.4) that

$$
\begin{aligned}
\liminf _{m \rightarrow \infty}\left|D \chi_{t}^{m}\right|(K) & \leqq \liminf _{m \rightarrow \infty} \int_{\Omega \times R} \psi(x, y)\left|D \chi_{t}^{m}\right| . \\
& \leqq \int_{\Omega \times R} \psi(x, y) d\|V(t)\| \\
& \leqq\|V(t)\|(B)
\end{aligned}
$$

Similarly, we show that

$$
\begin{aligned}
\int_{\Omega \times R} \psi(x, y) d\|V(t)\| & \leqq \limsup _{m \rightarrow \infty} \int_{\Omega \times R} \psi(x, y)\left|D \chi_{t}^{m}\right| \\
& \leqq \limsup _{m \rightarrow \infty}\left|D \chi_{t}^{m}\right|(B) .
\end{aligned}
$$

(6.13) and (6.14) proves (iii).

(iv) Let $B_{1}, B_{2}, \cdots$ be a sequence of open subsets of $\Omega \times \boldsymbol{R}$ satisfying $\bigcap_{k=1}^{\infty} B_{k}=\bar{B}$. Then we have from (iii) and (6.6) that

$$
\|V(t)\|\left(B_{k}\right) \geqq \liminf _{m \rightarrow \infty}\left|D \chi_{t}^{m}\right|(\bar{B}) \geqq \limsup _{m \rightarrow \infty}\left|D \chi_{t}^{m}\right|(B) \geqq\|V(t)\|(B),
$$

for $k=1,2, \cdots$, . As a consequence of the assumption (6.7), we see that $\lim _{k \rightarrow \infty}\|V(t)\|\left(B_{k}\right)=\|V(t)\|(B)$. It follows from this and (6.15) that $\lim _{m \rightarrow \infty}\left|D \chi_{t}^{m}\right|(B)$ $=\|V(t)\|(B)$. (iv) is proved.

Proposition 6.3. If $t \in \boldsymbol{R}_{3}$, then

$$
\left|D \chi_{t}\right|(B) \leqq\|V(t)\|(B)
$$


for any open subset $B \subset \Omega \times \boldsymbol{R}$. If for some $B \subset \Omega \times \boldsymbol{R}$

$$
\lim _{m \rightarrow \infty}\left|D \chi_{t}^{m}\right|(B)=\left|D \chi_{t}\right|(B),
$$

then

$$
\|V(t)\|(B)=\left|D \chi_{t}\right|(B) .
$$

Proof. Assume that $\phi(x, y) \in \mathcal{C}_{0}\left(B ; \boldsymbol{R}^{n+1}\right)$ and $|\psi(x, y)| \leqq 1$. Then from Proposition 6.2 , we have

$$
\begin{aligned}
\left|\int_{B} \psi(x, y) D \chi_{t}\right| & =\liminf _{m \rightarrow \infty}\left|\int_{B} \psi(x, y) D \chi_{t}^{m}\right| \\
& \leqq \liminf _{m \rightarrow \infty} \int_{B}|\psi(x, y)|\left|D \chi_{t}^{m}\right| \\
& \leqq \int_{B}|\psi(x, y)| d\|V(t)\| \\
& \leqq\|V(t)\|(B)
\end{aligned}
$$

Taking supremum with respect to $\phi$, we have (6.16).

If (6.17) holds, then

$$
\left|D \chi_{t}\right|(B) \leqq\|V(t)\|(B) \leqq \limsup _{m \rightarrow \infty}\left|D \chi_{t}^{m}\right|(B)=\left|D \chi_{t}\right|(B) .
$$

(6.18) holds in this case.

Proposition 6.4. There exists a subset $\boldsymbol{R}_{4} \subset \boldsymbol{R}$ such that $L_{1}\left(\boldsymbol{R} \backslash \boldsymbol{R}_{4}\right)=0$ and

$$
\int_{\Omega \times R} \psi(x, y) D_{n+1} \chi_{t}=\int_{\Omega \times R} \psi(x, y) d \| V(t)\left\llcorner\nu_{n+1} \|\right.
$$

for $t \in \boldsymbol{R}_{4}$ and $\phi \in \mathcal{C}_{0}(\Omega \times \boldsymbol{R})$. In particular, for any $t \in \boldsymbol{R}_{4}$ and $\phi \in \mathcal{C}_{0}(\Omega)$, we have

$$
-\int_{\Omega} \phi(x) d x=\int_{\Omega} \phi(x) d \| V(t)\left\llcorner\nu_{n+1} \| .\right.
$$

Proof. Let $\left\{\xi_{k}(x, y)\right\}_{k=1}^{\infty}$ be a countable dense subset of $\mathcal{C}_{0}(\Omega \times \boldsymbol{R})$. Then Proposition 5.1 asserts that for any $\xi_{k}$ and $t \in \boldsymbol{R}$

$$
\lim _{m \rightarrow \infty} \int_{\Omega \times \boldsymbol{R}} \xi_{k}(x, y) D_{n+1} \chi_{t}^{m}=\int_{\Omega \times \boldsymbol{R}} \xi_{k}(x, y) D_{n+1} \chi_{t} .
$$

Let $\phi \in L^{1}(\boldsymbol{R})$. Then multiplying $(6.22)$ by $\phi(t)$ and integrating with respect to $t$, we have 


$$
\begin{aligned}
& \int_{R} \phi(t) d t \int_{\Omega \times \boldsymbol{R}} \xi_{k}(x, y) D_{n+1} \chi_{t} \\
& \quad=\lim _{m \rightarrow \infty} \int_{\boldsymbol{R}} \phi(t) d t \int_{\Omega \times \boldsymbol{R}} \xi_{k}(x, y) D_{n+1} \chi_{t}^{m} \\
& \quad=\lim _{m \rightarrow \infty} \int_{\boldsymbol{R}} \phi(t) d t \int_{\Omega \times \boldsymbol{R} \times G} \xi_{k}(x, y) \nu_{n+1}(S) d V^{m}(t ; x, y, S) .
\end{aligned}
$$

Applying Proposition 4.3 to the right hand side of (6.23), we have

$$
\begin{aligned}
& \int_{\boldsymbol{R}} \phi(t) d t \int_{\Omega \times \boldsymbol{R}} \xi_{k}(x, y) D_{n+1} \chi_{t} \\
& \quad=\int_{\boldsymbol{R}} \phi(t) d t \int_{\Omega \times \boldsymbol{R} \times G} \xi_{k}(x, y) \nu_{n+1}(S) d V(t ; x, y, S) .
\end{aligned}
$$

Therefore there exists a subset $\boldsymbol{R}_{4} \subset \boldsymbol{R}$ such that $L_{1}\left(\boldsymbol{R} \backslash \boldsymbol{R}_{4}\right)=0$ and

$$
\int_{\Omega \times \boldsymbol{R}} \xi_{k}(x, y) D_{n+1} \chi_{t}=\int_{\Omega \times R \times G} \xi_{k}(x, y) \nu_{n+1}(S) d V(t ; x, y, S)
$$

for $k=1,2, \cdots$, and $t \in \boldsymbol{R}_{4}$. Since $\left\{\xi_{k}\right\}_{k}$ is dense in $\mathcal{C}_{0}(\Omega \times \boldsymbol{R})$, this proves (6.20) for any $\phi \in \mathcal{C}_{0}(\Omega \times R)$.

If $\phi \in \mathcal{C}_{0}(\Omega)$, then

$$
\int_{\Omega} \phi(x) D_{n+1} \chi_{t}=\lim _{m \rightarrow \infty} \int_{\Omega} \phi(x) D_{n+1} \chi_{t}^{m}=-\int_{\Omega} \phi(x) d x .
$$

This together with (6.20) proves (6.21).

Proposition 6.5. Let $\left(t_{0}, t_{1}\right)$ be an open interval and $B$ be an open subset of $\Omega \times \boldsymbol{R}$. Assume that

$$
\int_{B \times 1 \mathrm{rr}(G)} d V(t ; x, y, S)=0 \quad \text { for all } t \in\left(t_{0}, t_{1}\right) .
$$

Then there exists a subset $N \subset\left(t_{0}, t_{1}\right)$ such that $L_{1}(N)=0$ and

$$
\int_{\Omega \times R} \psi(x, y) D_{\jmath} \chi_{t}=\int_{\Omega \times R} \psi(x, y) d \| V(t)\left\llcorner\nu_{j} \|, \quad \jmath=1,2, \cdots, n,\right.
$$

for any $t \in\left(t_{0}, t_{1}\right) \backslash N$ and $\phi \in C_{0}(B)$.

Proof. Let $\left\{\xi_{k}\right\}_{k}$ be a countable dense subset of $\mathcal{C}_{0}(B)$. Let

$$
I_{j k}(t)=\int_{B} \xi_{k}(x, y) d \| V(t)\left\llcorner\nu_{j} \| .\right.
$$

Then (6.24) implies that

$$
I_{j k}(t)=\lim _{\varepsilon \rightarrow 0} I_{j k}^{\varepsilon}(t)
$$


where

$$
I_{j k}^{\varepsilon}(t)=\int_{B} \xi_{k}(x, y) \nu_{j}(S) \zeta_{\varepsilon}\left(\nu_{n+1}(S)\right) d V(t ; x, y, S)
$$

and $\zeta_{\varepsilon}(\tau)=1$ for $|\tau| \geqq \varepsilon$ and $\zeta_{\varepsilon}(\tau)=\varepsilon^{-1}|\tau|$ for $\varepsilon \geqq|\tau| \geqq 0$. Since $\nu_{j}(S) \zeta_{\varepsilon}\left(\nu_{n+1}(S)\right)$ is a continuous function of $S$, we can apply Proposition 4.3 to $I_{j k}^{\varepsilon}(t)$. Hence for any $\phi \in L^{1}\left(t_{0}, t_{1}\right)$ we have

$$
\begin{aligned}
\int_{t_{0}}^{t_{1}} \phi(t) I_{j k}(t) d t & =\lim _{\varepsilon \rightarrow 0} \lim _{m \rightarrow \infty} \int_{t_{0}}^{t_{1}} \phi(t) d t \int_{B \times G} \xi_{k}(x, y) \nu_{j}(S) \zeta_{\varepsilon}\left(\nu_{n+1}(S)\right) d V^{m}(t ; x, y, S) \\
& =\lim _{m \rightarrow \infty} \int_{t_{0}}^{t_{1}} \phi(t) d t \int_{B \times G} \xi_{k}(x, y) \nu_{j}(S) d V^{m}(t ; x, y, S)+\lim _{\varepsilon \rightarrow 0} \lim _{m \rightarrow \infty} J_{j k m}^{\varepsilon},
\end{aligned}
$$

where

$$
J_{j k m}^{\varepsilon}=\int_{t_{0}}^{t_{1}} \phi(t) d t \int_{B \times G} \xi_{k}(x, y) \nu_{j}(S)\left\{\zeta_{\varepsilon}\left(\nu_{n+1}(S)\right)-1\right\} d V^{m}(t ; x, y, S) .
$$

Using Proposition 5.1, we have

$$
\begin{aligned}
& \lim _{m \rightarrow \infty} \int_{t_{0}}^{t_{1}} \phi(t) d t \int_{B \times G} \xi_{k}(x, y) \nu_{j}(S) d V^{m}(t ; x, y, S) \\
& =\lim _{m \rightarrow \infty} \int_{t_{0}}^{t_{1}} \phi(t) d t \int_{B} \xi_{k}(x, y) D_{j} \chi_{t}^{m} \\
& =\int_{t_{0}}^{t_{1}} \phi(t) d t \int_{B} \xi_{k}(x, y) D_{j} \chi_{t} .
\end{aligned}
$$

On the other hand,

$$
\begin{aligned}
\lim _{m \rightarrow \infty}\left|J_{j k m}^{\varepsilon}\right| & \leqq \lim _{m \rightarrow \infty} \int_{t_{0}}^{t_{1}}|\phi(t)| d t \int_{B \times G}\left|\xi_{k}(x, y)\right|\left\{1-\zeta_{\varepsilon}\left(\nu_{n+1}(S)\right)\right\} d V^{m}(t ; x, y, S) \\
& \leqq \int_{t_{0}}^{t_{1}}|\phi(t)| d t \int_{B \times G}\left|\xi_{k}(x, y)\right|\left\{1-\zeta_{\varepsilon}\left(\nu_{n+1}(S)\right)\right\} d V(t ; x, y, S) .
\end{aligned}
$$

Therefore using (6.26), (6.27) and (6.28), we have

$$
\begin{aligned}
& \left|\int_{t_{0}}^{t_{1}} \phi(t) I_{j k}(t) d t-\int_{t_{0}}^{t_{1}} \phi(t) d t \int_{B} \xi_{k}(x, y) D_{j} \chi_{t}\right| \\
& \quad \leqq \limsup _{\varepsilon \rightarrow \infty} \int_{t_{0}}^{t_{1}}|\phi(t)| d t \int_{B \times G}\left|\xi_{k}(x, y)\right|\left\{1-\zeta_{\varepsilon}\left(\nu_{n+1}(S)\right)\right\} d V(t ; x, y, S) \\
& \quad=0
\end{aligned}
$$

because of (6.24). Since $\phi(t)$ is arbitrary, there exists a subset $N_{k}$ of $\boldsymbol{R}$ of $L_{1}$ measure 0 such that

$$
\int_{B} \xi_{k}(x, y) d \| V(t)\left\llcorner\nu_{j} \|=\int_{B} \xi_{k}(x, y) D_{\jmath} \chi_{t}, \quad k=1,2, \cdots, n,\right.
$$


for any $t \in\left(t_{0}, t_{1}\right) \backslash N_{k}$. Since $\left\{\xi_{k}\right\}_{k}$ is dense in $\mathcal{C}_{0}(B)$, we have (6.25) for any $\phi \in \mathcal{C}_{0}(B)$ and for $t \in\left(t_{0}, t_{1}\right) \backslash \bigcup_{k} N_{k}$.

We can state relationship of $\operatorname{spt} V(t ; x, y, S)$ and the graph of $u(t, x)$. Let $\pi: \Omega \times \boldsymbol{R} \times G \rightarrow \Omega \times \boldsymbol{R}$ be the projection. We call the set

$$
\operatorname{irr}(V(t))=\pi(\operatorname{spt} V(t) \cap \Omega \times \boldsymbol{R} \times \operatorname{irr}(G))
$$

the set of irregularity of $V(t)$. In this terminology we can see from propositions above the following

THEOREM 2. $\pi($ spt $V(t)) \backslash \operatorname{irr}(V(t)) \subset \operatorname{spt}\left|D \chi_{t}\right| \subset \partial^{*} E(t)$.

Proof of this Theorem is clear from Proposition 6.4 and 6.5.

The next proposition gives the direct relationship of the varifold $V(t ; x, y, S)$ and the graph of $u(t, x)$. We denote by $B(x, \rho)$ the open ball of radius $\rho>0$ centered at $x$ in $\Omega$.

Proposition 6.6. Let $V(t ; x, y, S)$ be the varifold solution of $\S 4$ and $u(t, x)$ be as above. Then at $L_{n+1}$-almost all $(t, x) \in \boldsymbol{R} \times \Omega$,

$$
u(t, x)=\lim _{\rho \rightarrow 0} u_{\rho}(t, x)
$$

where

$$
u_{\rho}(t, x)=\frac{-\int_{B(x, \rho) \times R} y d \| V(t)\left\llcorner\nu_{n+1} \|\right.}{L_{n}(B(x, \rho))} .
$$

Proof. Let $\mu$ be the Radon measure on $\Omega$ defined by the equality

$$
\mu(B)=\int_{B} u(t, x) d x .
$$

Then we have, at $L_{n}$-almost all $x$,

$$
u(t, x)=\lim _{\rho \rightarrow 0} \mu(B(x, \rho)) / L_{n}(B(x, \rho)) .
$$

On the other hand, for any $\phi(x) \in \mathcal{C}_{0}(\Omega)$, we have from Proposition 6.4 that

$$
\begin{aligned}
\int_{\Omega} u(t, x) \psi(x) d x & =\lim _{m \rightarrow \infty} \int_{\Omega} u^{m}(t, x) \psi(x) d x \\
& =-\lim _{m \rightarrow \infty} \int_{\Omega \times R} y \psi(x) D_{n+1} \chi_{t}^{m} \\
& =-\int_{\Omega \times R} y \psi(x) D_{n+1} \chi_{t} \\
& =-\int_{\Omega \times R} y \psi(x) d \| V(t)\left\llcorner\nu_{n+1} \| .\right.
\end{aligned}
$$


This means that

$$
\mu(B(x, \rho))=-\int_{B(x, \rho) \times R} y d \| V(t)\left\llcorner\nu_{n+1} \| .\right.
$$

Combining (6.32) and (6.33), we have (6.30) and (6.31).

As a consequence of Proposition 6.6 we may think that $u(t, x)$ represents the position of the membrane described by the varifold $V(t ; x, y, S)$. Therefore

$$
\frac{1}{2} \int_{\Omega}\left|D_{t} u(t, x)\right|^{2} d x
$$

represents the energy of motion. Similarly we can consider

$$
\int_{\Omega \times \boldsymbol{R} \times G} d V(t ; x, y, S)-|\Omega|
$$

as the potential energy.

Theorem 3. (Energy inequality). Let $u(t, x)$ be as in Proposition 5.1. Then $D_{t} u(t, *) \in L^{2}(\Omega)$ for $L_{1}$-almost every $t$ and we have

$$
\frac{1}{2} \int_{\Omega}\left|D_{t} u(t, x)\right|^{2} d x+\int_{\Omega \times \boldsymbol{R} \times G} d V(t ; x, y, S) \leqq M,
$$

where $M$ is as in Proposition 4.9. If $u_{0} \in W^{2+n / 2,2}(\Omega) \cap W_{0}^{1,2}(\Omega)$, then we have

$$
\begin{aligned}
& \frac{1}{2} \int_{\Omega}\left|D_{t} u(t, x)\right|^{2} d x+\int_{\Omega \times R \times G} d V(t ; x, y, S) \\
& \quad \leqq \frac{1}{2} \int_{\Omega}\left|u_{1}(x)\right|^{2} d x+\int_{\Omega}\left(1+\left|D u_{0}(x)\right|^{2}\right)^{1 / 2} d x .
\end{aligned}
$$

Proof. Using (4.8), we have

$$
\begin{aligned}
& \frac{1}{2} \int_{\Omega}\left|D_{t} u(t, x)\right|^{2} d x+\|V(t)\|(\Omega \times \boldsymbol{R}) \\
& \quad \leqq \frac{1}{2} \int_{\Omega}\left|D_{t} u^{m}(t, x)\right|^{2} d x+\limsup _{m \rightarrow \infty}\left|D \chi_{t}^{m}\right|(\Omega \times \boldsymbol{R}) \\
& \quad \leqq M
\end{aligned}
$$

If $u_{0}(x)$ is of class $W^{2+n / 2,2}(\Omega) \cap W_{0}^{1,2}(\Omega)$, then Sobolev's imbeding theorem asserts that $D P_{m} u_{0}(x)$ converges to $D u_{0}(x)$ uniformly. This yields that

$$
\lim _{m \rightarrow \infty} \int_{\Omega}\left(1+\left|D P_{m} u_{0}(x)\right|^{2}\right)^{1 / 2} d x=\int_{\Omega}\left(1+\left|D u_{0}(x)\right|^{2}\right)^{1 / 2} d x .
$$

Applying this to (4.7), we can prove (6.37). 
Next we prove

Lemma 6.7. Let $B$ be an open subset of $\Omega \times \boldsymbol{R}$. Assume that for $L_{1}$-almost all $t \in\left(t_{0}, t_{1}\right)$

$$
\left|D \chi_{t}\right|(B)=\lim _{m \rightarrow \infty}\left|D \chi_{t}^{m}\right|(B)
$$

Assume further that

$$
\int_{B \times 1 \mathrm{rr}(G)} d V(t ; x, y, S)=0 \quad \text { for almost all } t \in\left(t_{0}, t_{1}\right) .
$$

Then at almost all $t \in\left(t_{0}, t_{1}\right)$, the varifold $V(t ; x, y, S)$ is canonically identified with the function $u(t, x)$ in $B$. Let $\omega$ be an open subset of $\Omega$. Assume that (6.40) and (6.41) hold for $B=\omega \times \boldsymbol{R}$. Then $u(t, x)$ is a $B V$-solution of (1.1) in $\left(t_{0}, t_{1}\right) \times \omega$.

Proof. We have only to prove the first part of the Proposition. We put

$$
B(x, y ; \rho)=\left\{(w, z) \in \Omega \times \boldsymbol{R}:|w-x|^{2}+|z-y|^{2}<\rho^{2}\right\} .
$$

For any continuous function $\alpha(S)$ of $S \in G$, we consider

$$
V_{t}^{x, y}(\alpha)=\lim _{\rho \rightarrow 0} \int_{B(x, y, \rho)} \alpha(S) d V(t ; x, y, S) /\|V(t)\|(B(x, y ; \rho))
$$

for almost all $t$. This exists at $\|V(t)\|$-almost every $(x, y)$. (cf. 3.3 of Allard [1].)

The mapping $\mathcal{C}(G) \ni \alpha \rightarrow V_{t}^{x, y}(\alpha) \in \boldsymbol{R}$ defines a positive Radon measure $V_{t}^{x, y}(S)$ on $G$, that is,

$$
V_{t}^{x, y}(\alpha)=\int_{G} \alpha(S) d V_{t}^{x, y}(S)
$$

It is clear from the definition that

$$
\int_{G} d V_{t}^{x, y}(S)=1
$$

and that for any $\phi \in \mathcal{C}_{0}(\Omega \times \boldsymbol{R})$

$$
\begin{aligned}
& \int_{\Omega \times \boldsymbol{R} \times G} \psi(x, y) \alpha(S) d V(t ; x, y, S) \\
& \quad=\int_{\Omega \times \boldsymbol{R}} \psi(x, y)\left(\int_{G} \alpha(S) d V_{t}^{x, y}(S)\right) d\|V(t)\| .
\end{aligned}
$$

We cannot apply (6.45) to $\alpha(S)=\nu_{\jmath}(S), \jmath=1,2, \cdots, n$, because $\nu_{j}(S)$ is not continuous on $G$. We claim that if $\operatorname{spt} \phi$ is contained in $B$, then equality

$$
\int_{B \times G} \psi(x, y) \nu_{j}(S) d V(t ; x, y, S)=\int_{B} \psi(x, y)\left\{\int_{G} \tilde{\nu}_{j}(S) d V_{t}^{x, y}(S)\right\} d\|V(t)\|
$$


holds, where $\tilde{\nu}_{j}(S)=\nu_{j}(S)$ for $S \in G \backslash \operatorname{irr}(G)$ and $\tilde{\nu}_{j}(S)=0$ for $S \in \operatorname{irr}(G)$.

We prove the claim. Let $\varepsilon$ be an arbitrary positive number and $\zeta_{s}(t)$ be the function used in the proof of Proposition 6.5. Then

$$
\begin{aligned}
& \int_{B \times G} \psi(x, y) \nu_{j}(S) d V(t ; x, y, S) \\
& \quad=\int_{B \times G} \psi(x, y) \tilde{\nu}_{\jmath}(S) d V(t ; x, y, S) \\
& \quad=\lim _{s \rightarrow 0} \int_{B \times G} \psi(x, y) \nu_{j}(S) \zeta_{\varepsilon}\left(\nu_{n+1}(S)\right) d V(t ; x, y, S) .
\end{aligned}
$$

Since $\nu_{j}(S) \zeta_{\varepsilon}\left(\nu_{n+1}(S)\right)$ is a continuous function of $S$, we can apply (6.45) to the right hand side of (6.47). Thus we have

$$
\begin{aligned}
& \int_{B \times G} \phi(x, y) \nu_{j}(S) d V(t ; x, y, S) \\
& \quad=\lim _{s \rightarrow 0} \int_{B} \psi(x, y)\left(\int_{G} \nu_{j}(S) \zeta_{\varepsilon}\left(\nu_{n+1}(S)\right) d V_{t}^{x, y}(S)\right) d\|V(t)\| \\
& \quad=\int_{B} \psi(x, y)\left(\int_{G} \tilde{\nu}_{j}(S) d V_{t}^{x, y}(S)\right) d\|V(t)\| .
\end{aligned}
$$

We have proved the claim (6.46).

Next we wish to prove that

$$
\nu_{\jmath}(t ; x, y)=\int_{G} \tilde{\nu}_{j}(S) d V_{t}^{x, y}(S), \quad \jmath=1,2, \cdots, n+1,
$$

for almost all $t$ and $\|V(t)\|$-almost every $(x, y) \in B$. In fact combining Proposition 6.5 and (6.46), we have

$$
\begin{aligned}
& \int_{B} \phi(x, y) \nu_{\jmath}(t ; x, y)\left|D \chi_{t}\right|=\int_{B} \psi(x, y) D_{\jmath} \chi_{t} \\
& =\int_{B} \phi(x, y) d\left\|V(t) L_{\nu_{\jmath}}\right\| \\
& =\int_{B \times G} \phi(x, y) \nu_{j}(S) d V(t ; x, y, S) \\
& =\int_{B} \phi(x, y)\left\{\int_{G} \tilde{\nu}_{j}(S) d V_{t}^{x, y}(S)\right\} d\|V(t)\| .
\end{aligned}
$$

As a consequence of (6.49), for any $(x, y) \in B$ and for sufficiently small $\rho>0$, we have 


$$
\begin{aligned}
& \int_{B(x, y ; \rho)} \nu_{j}(t ; x, y)\left|D \chi_{t}\right| \\
& \quad=\int_{B(x, y ; \rho)}\left(\int_{G} \tilde{\nu}_{j}(S) d V_{t}^{x, y}(S)\right) d\|V(t)\| .
\end{aligned}
$$

For each $(x, y) \in B$ and almost all $t$, we can choose a sequence of positive numbers $\left\{\rho_{k}\right\}_{k=1}^{\infty}$, such that

$$
\lim _{k \rightarrow \infty} \rho_{k}=0
$$

and

$$
\|V(t)\|\left(\partial B\left(x, y ; \rho_{k}\right)\right)=0, \quad k=1,2, \cdots, .
$$

By virtue of Proposition 6.3 and (6.52), we have

$$
\left|D \chi_{t}\right|\left(\partial B\left(x, y ; \rho_{k}\right)\right)=0, \quad k=1,2, \cdots, .
$$

This and assumption (6.40) imply that

$$
\left|D \chi_{t}\right|\left(B\left(x, y ; \rho_{k}\right)\right)=\lim _{m \rightarrow \infty}\left|D \chi_{t}^{m}\right|\left(B\left(x, y ; \rho_{k}\right)\right)
$$

(cf. Giusti [6]). Using Proposition 6.2 (iv), we see that

$$
\left|D \chi_{t}\right|\left(B\left(x, y ; \rho_{k}\right)\right)=\|V(t)\|\left(B\left(x, y ; \rho_{k}\right)\right), \quad k=1,2, \cdots, .
$$

This together with (6.50) yields that

$$
\begin{aligned}
& \int_{B\left(x, y ; \rho_{k}\right)} \nu_{j}(t ; x, y)\left|D \chi_{t}\right| /\left|D \chi_{t}\right|\left(B\left(x, y ; \rho_{k}\right)\right) \\
& \quad=\int_{B\left(x, y, \rho_{k}\right)}\left(\int_{G} \tilde{\nu}_{j}(S) d V_{t}^{x, y}(S)\right) d\|V(t)\| /\|V(t)\|\left(B\left(x, y ; \rho_{k}\right)\right) .
\end{aligned}
$$

Let $k$ tend to $\infty$ and take the limit of (6.54). Then (6.51) and Besicovitch's theorem (cf. [3] or [5]) give (6.48).

Applying the next Lemma 6.8 to (6.48), we conclude that

$$
\tilde{\nu}_{j}(S)=\nu_{j}(t ; x, y)
$$

at $V_{t}^{x, y}$-almost all $S \in G$. If $S \neq S^{\prime}$ then $\tilde{\nu}_{j}(S) \neq \tilde{\nu}_{j}\left(S^{\prime}\right)$. Thus (6.55) implies that

$$
\text { spt } V_{t}^{x, y}=\text { one point }=\operatorname{Tan}_{x, y} \partial^{*} E(t) .
$$

And for each $\alpha \in C(G)$, we have

$$
\int_{G} \alpha(S) d V_{t}^{x, y}(S)=\alpha\left(\operatorname{Tan}_{x, y} \partial^{*} E(t)\right) .
$$

It follows from (6.56), (6.45), (6.53) and Besicovitch's theorem that for any $\phi \in \mathcal{C}_{0}(B \times G)$, we have 


$$
\begin{aligned}
& \int_{B \times G} \phi(x, y, S) d V(t ; x, y, S) \\
& \quad=\int_{B \times G} \psi\left(x, y, \operatorname{Tan}_{x, y} \partial^{*} E(t)\right) d\|V(t)\| \\
& =\int_{B \times G} \psi\left(x, y, \operatorname{Tan}_{x, y} \partial^{*} E(t)\right)\left|D \chi_{t}\right| .
\end{aligned}
$$

Therefore $V(t ; x, y, S)$ is canonically identified with the graph of $u(t, x)$. Lemma 6.7 has been proved upto the following Lemma 6.8.

LeMma 6.8. Let $P$ be a probability measure on a space $X$. Let $v(x)$ be an $\boldsymbol{R}^{n}$-valued function which is integrable with respect to $P$. Let

$$
v=\int_{X} v(x) d P(x) .
$$

Assume that $|v(x)| \leqq 1$ and $|v|=1$. Then $v=v(x)$ at P-almost every $x$.

Proof is clear.

THEOREM 4. Assume that $u_{1} \in L^{2}(\Omega)$ and $u_{0} \in W^{2+n / 2,2}(\Omega)$. Assume further that the function $u(t, x)$ of Proposition 5.1 satisfies the energy conservation law for $t \in\left(t_{0}, t_{1}\right)$, i.e.,

$$
\begin{aligned}
& \frac{1}{2} \int\left|D_{t} u(t, x)\right|^{2} d x+\int_{\Omega \times R}\left|D \chi_{t}\right| \\
& \quad=\frac{1}{2} \int_{\Omega}\left|u_{1}(x)\right|^{2} d x+\int_{\Omega}\left(1+\left|D u_{0}(x)\right|^{2}\right)^{1 / 2} d x .
\end{aligned}
$$

Let $\omega$ be any open subset of $\Omega$ such that

$$
\int_{\omega \times \boldsymbol{R} \times \operatorname{1rr}(G)} d V(t ; x, y, S)=0
$$

for atmost all $t \in\left(t_{0}, t_{1}\right)$. Then at $L_{1}$-almost all $t \in\left(t_{0}, t_{1}\right)$, the varifold solution $V(t ; x, y, S)$ is canonically identified with the graph of the function $u(t, x)$ at $\mathcal{H}_{n+1}$-almost every $(x, y) \in \omega \times \boldsymbol{R}$ and $u(t, x)$ is the solution of $(1.1)$ in $\left(t_{0}, t_{1}\right) \times \omega$.

Proof. Let

$$
M_{m}=\frac{1}{2} \int_{\Omega}\left|P_{m} u_{1}(x)\right|^{2} d x+\int_{\Omega}\left(1+\left|D P_{m} u_{0}(x)\right|^{2}\right)^{1 / 2} d x .
$$

Then the proof of (6.37) asserts that

$$
\lim _{m \rightarrow \infty} M_{m}=\frac{1}{2} \int_{\Omega}\left|u_{1}(x)\right|^{2} d x+\int_{\Omega}\left(1+\left|D u_{0}(x)\right|^{2}\right)^{1 / 2} d x .
$$

We have from (4.7) 


$$
M_{m}=\frac{1}{2} \int_{\Omega}\left|D_{t} u^{m}(t, x)\right|^{2} d x+\int_{\Omega \times R}\left|D \chi_{t}^{m}\right| .
$$

The assumption (6.58) means that

$$
\begin{aligned}
& \frac{1}{2} \int_{\Omega}\left|D_{t} u(t, x)\right|^{2} d x+\int_{\Omega \times \boldsymbol{R}}\left|D \chi_{t}\right| \\
& \quad=\lim _{m \rightarrow \infty}\left\{\frac{1}{2} \int_{\Omega}\left|D_{t} u^{m}(t, x)\right|^{2} d x+\int_{\Omega \times \boldsymbol{R}}\left|D \chi_{t}^{m}\right|\right\} .
\end{aligned}
$$

Since

$$
\begin{aligned}
& \int_{\Omega}\left|D_{t} u(t, x)\right|^{2} d x \leqq \limsup _{m \rightarrow \infty} \int_{\Omega}\left|D_{t} u^{m}(t, x)\right|^{2} d x \\
& \int_{\Omega \times \boldsymbol{R}}\left|D \chi_{t}\right| \leqq \limsup _{m \rightarrow \infty} \int_{\Omega \times \boldsymbol{R}}\left|D \chi_{t}^{m}\right|,
\end{aligned}
$$

the equality (6.62) asserts that equalities hold in both (6.63) and (6.64), namely, we have

$$
\int_{\Omega}\left|D_{t} u(t, x)\right|^{2} d x=\lim _{m \rightarrow \infty} \int_{\Omega}\left|D_{t} u^{m}(t, x)\right|^{2} d x
$$

and

$$
\int_{\Omega \times R}\left|D \chi_{t}\right|=\lim \int_{\Omega \times R}\left|D \chi_{t}^{m}\right|
$$

Therefore, the set $\Omega \times \boldsymbol{R}$ itself satisfies the condition (6.40) of Lemma 6.7. As the consequence of Lemma 6.7, we can prove Theorem 4 .

\section{§ 7. Generalized Hamilton's principle.}

So far we have treated the special varifold solution $V(t ; x, y, S)$ constructed in $\S 4$. In the present section we treat any varifold solution $W(t ; x, y, S)$ of (1.1) satisfying additional conditions which will be given below. And we prove that a generalized Hamilton's principle holds for such a good varifold solution.

We define measures $\| W(t)\left\llcorner\nu_{j} \|, \jmath=1,2, \cdots, n+1\right.$, on $\Omega \times \boldsymbol{R}$ by the following formula: For any Borel set $A \subset \Omega \times \boldsymbol{R}$

$$
\| W(t)\left\llcorner\nu_{\jmath} \|(A)=\int_{A \times G} \nu_{j}(S) d W(t ; x, y, S)\right.
$$

in just the same way as in $\S 6$. In analogy with Proposition 6.6, we put, for $x \in \Omega$ and $t \in \boldsymbol{R}$,

$$
w(t, x)=\lim _{\rho \rightarrow 0} w_{\rho}(t, x),
$$

where 


$$
w_{\rho}(t, x)=\int_{B(x, \rho) \times \boldsymbol{R}} y d \| W(t)\left\llcorner\nu _ { n + 1 } \| / \int _ { B ( x , \rho ) \times \boldsymbol { R } } d \| W ( t ) \left\llcorner\nu_{n+1} \| .\right.\right.
$$

We call $w(t, x)$ the position of the membrane. It follows from Besicovitch's theorem that $w(t, x)$ exists almost every $x$ with respect to the measure $\| W(t)\left\llcorner_{n+1} \|\right.$.

We call

$$
\frac{1}{2} \int_{\Omega}\left|D_{t} w(t, x)\right|^{2} d x
$$

the energy of motion if it is finite. Similarly, we may call

$$
\int_{\Omega \times \boldsymbol{R} \times G} d W(t ; x, y, S)-|\Omega|=\int_{\Omega \times \boldsymbol{R}} d\|W(t)\|-|\Omega|
$$

the potential energy.

We assume that the following conditions hold for the varifold solution $W(t ; x, y, S)$ :

(A1) The position function $w(t, x)$ is a function of bounded variation in $\Omega$ for a fixed $t \in \boldsymbol{R}$ and spt $\| W(t)\left\llcorner\nu_{n+1} \| \subset \partial^{*} F(t)\right.$, where $F(t)$ is the subgraph of the function $w(t, x)$.

(A2) $D_{t} w(t, x) \in L^{2}(\Omega)$ for each $t$ and

$$
\int_{0}^{T} d t \int_{\Omega} \frac{1}{2}\left|D_{t} w(t, x)\right|^{2} d x+\int_{0}^{T} d t \int_{\Omega \times R \times G} d W(t ; x, y, S)<\infty .
$$

(A3) For each $\phi(x) \in \mathcal{C}_{0}(\Omega)$

$$
-\int_{\Omega \times \boldsymbol{R}} \psi(x) d \| W(t)\left\llcorner_{\nu_{n+1}} \|=\int_{\Omega} \psi(x) d x .\right.
$$

The last equality expresses a generalization of the law of conservation of mass. As we have proved in $\S 6$, the varifold solution $V(t ; x, y, S)$ constructed in $\S 4$ has all these properties.

If $W(t ; x, y, S)$ satisfies all of these conditions, then we consider the action

$$
A(W)=\int_{0}^{T} d t \int_{\Omega} \frac{1}{2}\left|D_{t} w(t, x)\right|^{2} d x-\int_{0}^{T} d t\left\{\int_{\Omega \times \boldsymbol{R} \times G} d W(t ; x, y, S)-|\Omega|\right\},
$$

and we shall show that $W$ is a critical point of this action functional, i. e.,

$$
\delta A(W)=0 \text {. }
$$

To state this fact more precisely we introduce admissible functions $\psi(t, x)$ $\in \mathcal{C}^{2}(\boldsymbol{R} \times \Omega)$ such that

$$
\phi(0, x)=D_{t} \psi(0, x)=0, \quad \psi(T, x)=D_{t} \psi(T, x)=0
$$

and $\left.\phi(t, x)\right|_{\partial \Omega}=0$. Then for each $\sigma \in \boldsymbol{R}$ we can define a diffeomorphism

$$
\eta(\boldsymbol{\sigma}): \Omega \times \boldsymbol{R} \ni(x, y) \rightarrow(x, y+\sigma \phi(t, x)) \in \Omega \times \boldsymbol{R} .
$$


This induces a map $\eta(\sigma)_{\#}$ of varifolds, which is defined by the equality

$$
\begin{aligned}
& \left\langle\eta(\sigma)_{\#} W(t), \phi\right\rangle \\
& \quad=\int_{\Omega \times \boldsymbol{R} \times G} \psi(x, y+\sigma \psi(t, x), D \eta(\sigma) S)\left|\wedge^{n} D \eta(\sigma)\right| d W(t ; x, y, S) .
\end{aligned}
$$

(cf. Allard [1], §3.2), where $D \eta(\sigma)$ is the differential of the map $\eta(\sigma)$ and $\wedge^{n} D \eta(\sigma)$ is its $n$-exterior product. The precise formulation of the generalized Hamilton's principle is

THEOREM 5. Assume that $W(t ; x, y, S)$ is a varifold solution of the equations (1.1) and (1.2) and that it satisfies the assumptions (A1), (A2) and (A3). Then

$$
\left.\frac{d}{d \sigma} A\left(\eta(\sigma)_{\#} W\right)\right|_{\sigma=0}=0 \text {. }
$$

Proof. We first calculate the position $w^{\sigma}(t, x)$ corresponding to the varifold $\eta(\sigma)_{\#} W(t ; x, y, S)$, that is,

$$
w^{\sigma}(t, x)=\lim _{\rho \rightarrow 0} w_{\rho}^{\sigma}(t, x),
$$

where

$$
w_{\rho}^{\sigma}(t, x)=\frac{\int_{B(x, \rho) \times \boldsymbol{R} \times G} y \nu_{n+1}(S) d\left(\eta(\boldsymbol{\sigma})_{\sharp} W(t ; z, y, S)\right)}{\int_{B(x, \rho) \times \boldsymbol{R} \times G} \nu_{n+1}(S) d\left(\eta(\sigma)_{\sharp} W(t ; z, y, S)\right)} .
$$

We have for any $x \in \Omega$ and $\rho>0$,

$$
\begin{aligned}
& \int_{B(x, \rho) \times \boldsymbol{R} \times G} y \nu_{n+1}(S) d\left(\eta(\sigma)_{\#} W(t ; z, y, S)\right) \\
& \quad=\int_{B(x, \rho) \times \boldsymbol{R} \times G}(y+\sigma \psi(t, z)) \nu_{n+1}(D \eta(\sigma) S)\left|\wedge^{n} D \eta(\boldsymbol{\sigma})\right| d W(t ; z, y, S) .
\end{aligned}
$$

Using assumptions (A1) and (A3), we see that this is equal to

$$
\int_{B(x, \rho) \times R \times G}(w(t, z)+\sigma \psi(t, z)) \nu_{n+1}(D \eta(\sigma) S)\left|\wedge^{n} D \eta(\sigma)\right| d W(t ; z, y, S) .
$$

Similarly, we have

$$
\begin{aligned}
& \int_{B(x, \rho) \times R \times G} \nu_{n+1}(S) d\left(\eta(\sigma)_{\#} W(t ; z, y, S)\right) \\
& \quad=\int_{B(x, \rho) \times R \times G} \nu_{n+1}(D \eta(\sigma) S)\left|\wedge^{n} D \eta(\sigma)\right| d W(t ; z, y, S) .
\end{aligned}
$$

It follows from (7.11), (7.12), (7.13), (7.14) and Besicovitch's theorem that

$$
w^{\sigma}(t, x)=w(t, x)+\sigma \phi(t, x),
$$


at almost every $x \in \Omega$ with respect to the measure $\mu$ such that for any Borel subset $B \subset \Omega$

$$
\mu(B)=\int_{B \times \boldsymbol{R} \times G} \nu_{n+1}(D \eta(\sigma) S)\left|\wedge^{n} D \eta(\sigma)\right| d W(t ; x, y, S) .
$$

We claim that (7.16) holds at $L_{n}$-almost all $x$ in $\Omega$. To prove this we shall show that an $n$-dimensional vector subspace $S \in G$ satisfies $\nu_{n+1}(D \eta(\sigma) S)=0$ if and only if $\nu_{n+1}(S)=0$. Assume that $\nu_{n+1}(S) \neq 0$. Then we can choose a basis $v_{1}, v_{2}, \cdots, v_{n}$ of $S$ so that $v_{1}=e_{1}+\beta_{1} e_{n+1}, v_{2}=e_{2}+\beta_{2} e_{n+1}, \cdots, v_{n}=e_{n}+\beta_{n} e_{n+1}$, where $e_{2}, i=1,2, \cdots, n$, is the unit vector parallel to the $x_{i}$-axis and $e_{n+1}$ is the unit vector parallel to the $y$-axis. Since $D \eta(\sigma) v_{i}=e_{i}+\left(\beta_{i}+\sigma \frac{\partial}{\partial x_{\imath}} \psi(t, x)\right) e_{n+1}$, we have

$$
v_{1} \wedge v_{2} \wedge \cdots \wedge v_{n}=e_{1} \wedge e_{2} \cdots \wedge e_{n}+g \wedge e_{n+1}
$$

with some $g \in \wedge^{n-1} \boldsymbol{R}^{n}$. This implies that $\nu_{n+1}(D \eta(\sigma) S) \neq 0$. Similarly we can prove that $\nu_{n+1}(D \eta(\sigma) S)=0$ if $\nu_{n+1}(S)=0$.

Since $\left|\wedge^{n} D \eta(\sigma)\right|$ never vanishes, we see that

$$
0=\mu(B)=\int_{B \times \boldsymbol{R} \times G} \nu_{n+1}(D \eta(\sigma) S)\left|\wedge^{n} D \eta(\sigma)\right| d W(t ; x, y, S)
$$

if and only if

$$
L_{n}(B)=\int_{B \times R \times G} \nu_{n+1}(S) d W(t ; x, y, S)=0 .
$$

This proves that (7.16) holds for $L_{n}$-almost every $x$ in $\Omega$. Thus we have

$$
\begin{aligned}
A\left(\eta(\sigma)_{\#} W\right)= & \frac{1}{2} \int_{0}^{T} d t \int_{\Omega}\left|D_{t} w(t, x)+\sigma D_{t} \psi(t, x)\right|^{2} d x \\
& -\int_{0}^{T} d t \int_{\Omega \times R \times G} d\left(\eta(\sigma)_{\#} W(t ; x, y, S)\right)+|\Omega| .
\end{aligned}
$$

We now calculate the variation $\left.\frac{d}{d \sigma} A\left(\eta(\sigma)_{\#} W\right)\right|_{\sigma=0}$. First we have

$$
\begin{aligned}
& \left.\frac{d}{d \sigma} \int_{0}^{T} d t \int_{\Omega} \frac{1}{2}\left|D_{t} w^{\sigma}(t, x)\right|^{2} d x\right|_{\sigma=0} \\
& \quad=\left.\frac{d}{d \sigma} \int_{0}^{T} d t \int_{\Omega} \frac{1}{2}\left|D_{t} w(t, x)+\sigma \psi(t, x)\right|^{2} d x\right|_{\sigma=0} \\
& \quad=\int_{0}^{T} d t \int_{\Omega \times \boldsymbol{R} \times G} w(t, x) D_{t}^{2} \psi(t, x) \nu_{n+1}(S) d W(t ; x, y, S) \\
& \quad=\int_{0}^{T} d t \int_{\Omega \times \boldsymbol{R} \times G} D_{t}^{2} \psi(t, x) y \nu_{n+1}(S) d W(t ; x, y, S) .
\end{aligned}
$$

Next we describe the variation of the second term of the right hand side of 
(7.17). Let $\dot{\eta}(x, y)=\left.D_{\sigma} \eta(\sigma)(x, y)\right|_{\sigma=0}=(0,0, \cdots, \phi(t, x))$ be the vector field which is the tangent at $\sigma=0$ to the 1-parameter family of diffeomorphisms $\eta(\sigma)$. We know that (cf. Allard [1], §3.3)

$$
\begin{aligned}
& \left.\frac{d}{d \sigma} \int_{\Omega \times \boldsymbol{R} \times G} d\left(\eta(\sigma)_{\#} W\right)(t ; x, y, S)\right|_{\sigma=0} \\
& \quad=\int_{\Omega \times \boldsymbol{R} \times G} \sum_{k=1}^{n} D_{k} \psi(t, x) \nu_{k}(S) \nu_{n+1}(S) d W(t ; x, y, S) .
\end{aligned}
$$

Consequently

$$
\begin{aligned}
& \left.\frac{d}{d \sigma}\left(A\left(\eta(\sigma)_{\#} W\right)\right)\right|_{\sigma=0} \\
& =\int_{0}^{T} d t \int_{\Omega \times \boldsymbol{R} \times G} D_{t}^{2} \psi(t, x) y \nu_{n+1}(S) d W(t ; x, y, S) \\
& \quad+\int_{0}^{T} d t \int_{\Omega \times \boldsymbol{R} \times G} \sum_{k=1}^{n} D_{k} \psi(t, x) \nu_{k}(S) \nu_{n+1}(S) d W(t ; x, y, S) .
\end{aligned}
$$

Since $W(t ; x, y, S)$ is a varifold solution of $(1.1),(1.3)$ and $\phi(0, x)=D_{t} \psi(0, x)=0$, the right hand side vanishes by virtute of (3.3). We have

$$
\left.\frac{d}{d \sigma} A\left(\eta(\sigma)_{\#} W\right)\right|_{\sigma=0}=0 \text {. }
$$

Theorem 6 is proved.

\section{REFERENCES}

[1] W. K. Allard, On the first variation of a varifold, Ann. of Math. vol. 95 (1972), 417-491.

[2] F.J. Almgren, JR., The theory of varifolds, Mimeographed notes, Princeton (1965).

[3] A.S. Besicovitch, A general form of the covering principle and relative differentiation of additive functions I, Proc. Cambridge Phil. Soc. vol. 41 (1945), 103-110. II vol. 42 (1946), 1-10.

[4] E. De Giorgi, Su una teoria generale della misura $(r-1)$-dimendinale in uno spazio ad $r$ dimensioni, Ann. Mat. Pura Appl. (4) vol. 36 (1954), 191-213.

[5] R. J. DiPerna, Convergence of approximate solutions to conservation laws, Arch. Rational Mech. Anal. vol. 82 (1983), 27-70.

[6] H. Federer, Geometric measure theory, Springer-Verlag, New York, 1969.

[7] D. Fujiwara, A. Inoue and S. TAKakuwa, A varifold solution of nonlinear wave equation of a membrane, Proc. Japan Acad. Sci. vol. 60 (1984), 113-116.

[8] E. Giusti, Minimal surfaces and functions of bounded variation, Notes on Pure Mathematics 10, Australian National University, Canberra (1977).

[9] T. KAто, Linear and quasi-linear equations of evolution of hyperbolic type, C. I. M.E. vol. II (1976), 125-191.

[10] Y. Shibata AND Y. Tsutsumi, Local existence of $C^{\infty}$-solution for the initialboundary value problem for fully nonlinear wave equation, (preprint). 
[11] L. TARTAR, Une nouvelle méthode de résolution d'équations aux dérivées partielles nonlinéaires, Lecture Notes in Mathematics, vol. 564, 13-25.

[12] L. TARTAR, Compensated compactness and applications to partial differential equations, in Research Notes in Mathematics, Nonlinear analysis and mechanics . Heriot-Watt Symposium, vol. 4, ed. R. J. Knops, Pitman Press, (1979).

Department of Mathematics

TOKyo Institute of Technology

Oh-okayama, Meguro-ku, Tokyo 152

JAPAN
Department of Mathematics

Tokyo Metroporitan University

Fukasawa, Setagaya-ku, Tokyo 158

JAPAN 